\title{
Scalable Internet of Things Network Design Using Multi-hop IEEE 802.11ah
}

Nurzaman Ahmed ( $\nabla$ nurzaman713@gmail.com )

North-Eastern Hill University https://orcid.org/0000-0003-2597-6483

Md. Iftekahr Hussain

North-Eastern Hill University

\section{Research Article}

Keywords: IEEE 802.11ah, Sub-1 GHz, Restricted Access Window (RAW), Internet of Things (IoT), M2M communication, Relay AP

Posted Date: April 27th, 2021

DOl: https://doi.org/10.21203/rs.3.rs-241548/v1

License: (1) This work is licensed under a Creative Commons Attribution 4.0 International License. Read Full License 


\title{
Scalable Internet of Things Network Design Using Multi-hop IEEE 802.11ah
}

\author{
Nurzaman Ahmed • Md. Iftekhar Hussain
}

Received: date / Accepted: date

\begin{abstract}
The emerging IEEE 802.11ah is a promising communication standard for large-scale networks particularly the Internet of Things (IoT). The singlechannel-based centralized channel access mechanism employed in 802.11ah does not scale well in such networks and leads to poor data reception quality. In this paper, we propose a multi-band sectorization and dynamic load balancing scheme for improving scalability. These features facilitate multi-hop communication more efficiently and enhance network capacity. Traffic congestion issues prevailing around the access point node due to the large volume of uplink traffic is mitigated by allowing simultaneous transmission using multiple orthogonal channels and sectors. Simulation and analytical results establish the essence of the novel protocol by showing significant improvements in terms of throughput and average packet delay over the existing schemes. The proposed network architecture improves throughput and delay performance up to $150 \%$ and $100 \%$ respectively compared to the relevant schemes.
\end{abstract}

Keywords IEEE 802.11ah · Sub-1 GHz - Restricted Access Window (RAW) - Internet of Things (IoT) . M2M communication - Relay AP.

IEEE 802.11ah-based large-scale Internet of Things (IoT) provides poor network performance due to long communication range and higher delay in multi-hop transmission. As it uses a single channel, only one node in the network is allowed to transmit at a particular time. In a network with a large number of relay nodes and multi-hop links, uplink traffic forwarded by the end

N. Ahmed and Md. I. Hussain

Department of Information Technology, North-Eastern Hill University, Shillong, India-793022

E-mail: nurzaman713@nehu.ac.in,ihussain@nehu.ac.in stations (STAs) create congestion around the Access Point (AP). Such networks cannot support smooth and efficient communication which remains a key challenge for many IoT applications.

This paper proposes a scalable Medium Access Control (MAC) layer solution for the IEEE 802.11ah network. Our scheme maximizes the overall network performance by an adaptive sectorization and channel allocation mechanism. For supporting distribution channel access in terms of RAW scheduling among different relay nodes, the proposed architecture enables the nodes to contend and get associated with the AP in a distributed manner. Additionally, a dynamic bandwidth adjustment is incorporated at the relay nodes to improves channel efficiency and QoS requirements of IoT applications.

\subsection{Motivation}

The deployment of IoT is growing very fast, ranging its applications and services from the environment to healthcare [20]. The emerging IEEE 802.11ah technology has proven as a promising communication standard for IoT ([21] and [25]). To meet the technical requirements such as scalability, heterogeneity, and low power consumption, IEEE 802.11ah has come up with some novel ideas. It allows up to 8,191 devices to be associated with an Access Point (AP) by using a new hierarchical Association IDentification (AID) scheme. With the use of sub-1 GHz channel bands, it can cover up to $1 \mathrm{~km}$ in a single hop, which can further be extended by using relay nodes between the AP and stations (STAs) ([19] and [12]). IEEE 802.11ah adopts strategies like Target Wake Time (TWT), Traffic Indication Map (TIM) and Segmentation, and Restricted 


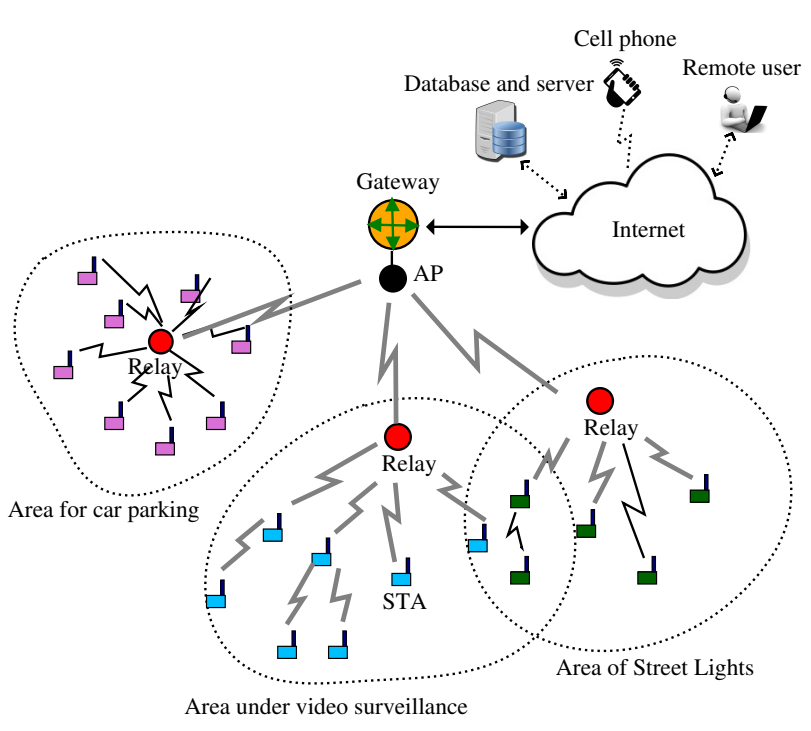

Fig. 1: IEEE 802.11ah-based smart city network architecture spreading over large coverage area

Access Window (RAW) for efficient communication in large-scale networks. It enables seamless communication among heterogeneous applications such as smart cities, industrial IoT, and smart healthcare. Figure 1 shows a network architecture for the smart city including a few possible IoT applications. Interconnection among various components like AP, Relay AP (RAP), end STAs, and the Internet backbone has been clearly depicted. The extended coverage range supported by RAP facilitates connectivity among multiple service regions such as car parking, street light control, and video surveillance in a multi-hop scenario.

Large-scale network architecture contains a huge number of relay nodes and multi-hop links. Uplink traffic forwarded by the end STAs create congestion towards the AP node. Due to the lack of parallel transmission facilities at AP and RAP nodes, such a heavy traffic region becomes a bottleneck in large scale networks. Further, IoT networks need to support heterogeneous traffic requirements in terms of packet interval and transmission behaviors. A real-time surveillance application requires higher data-are to transmit highquality videos. Severe hidden node problem in communication may also arise if the STAs are not grouped according to their geographic locations, especially in urban infrastructures. In such a dense network scenario, the overall network may lead to beggarly performance without spatial reuse of the channel. Many IoT applications require some sort of Quality of Service (QoS) guarantees in terms of parameters like delay and throughput. Further, an efficient RAP solution that considers the channel access and traffic requirements of STAs is also an open area of research.

The state-of-the-art works such as [23], [27], [3], [29], [24] and [28] improve scalability up to a certain extent. However, due to the traffic congestion and high channel access delay, the existing solutions are not adequate for enabling scalable communication in IoT. Dynamic bandwidth allocation schemes such as [18] and [17] are not sufficient for heterogeneous traffic demands of the groups and STAs. The growing traffic demands of IoT requires spatial reuse of channels and network capacity enhancement.

\subsection{Contribution}

In summary, the following are the key contributions of this work:

- We introduce a multi-band sectorization scheme for IEEE 802.11ah to combat the congestion issue and improve holistic network performance. The proposed solution uses Frequency-division multiplexing (FDM) at the AP node for supporting multiple sector antennas and allows concurrent transmissions using multiple channels.

- A MAC scheme is designed, which periodically checks the current load at the AP or RAPs nodes, and accordingly adjust bandwidth by switching to suitable Modulation and Coding Schemes (MCSs) dynamically. The congestion state of a RAP node is determined by measuring the current collision ratio.

- An efficient Transmission Opportunity (TXOP)-based scheduling scheme for AP and RAP communication is presented. The proposed MAC protocol switches its TXOP operation based on traffic patterns such as Uplink or Downlink.

\section{Background and Related Works}

To support the requirements of heterogeneous traffic in IoT, IEEE 802.11ah has proposed different MCSs to be used in various scenarios. For data transmission, 1, 2, 4, 7, and $16 \mathrm{MHz}$ channel bands are defined [9]. Considering a wider channel bandwidth (16 MHz), MCS9 can provide up to $78 \mathrm{Mbps}$ of data rate.

\subsection{Channelization}

The channelization scheme of IEEE 802.11ah inherits the PHY layer of 802.11ac. The available license-exempt channels in the sub-1 GHz spectrum depends on the country's regulation. For example, current availability 
of sub-1 GHz bands in different countries - 902-928 $\mathrm{MHz}$ (US), 755-787 \& 614-787 MHz (China), 917-923.5 $\mathrm{MHz}$ (South Korea), 863-868.6 MHz (Europe), 865-867 $\mathrm{MHz}$ (India), 915.9-929.7 MHz (Japan), 866-869 MHz, 920-925 \& 866-869 MHz (Singapore) [22]. The channel bandwidth in IEEE 802.11ah includes 1, 2, 4, 8, and $16 \mathrm{MHz}$, whereas 1 and $2 \mathrm{MHz}$ are widely available in many countries and more suitable for low rate traffic. In the United States, there are 26 numbers of $1 \mathrm{MHz}$ and 13 numbers of $2 \mathrm{MHz}$ channels.

\subsection{Relay Node Support}

A relay node that is positioned between the AP and end nodes extends connectivity to the STAs located outside AP's coverage. IEEE 802.11ah implements RAP consisting of an AP and a STA module in it [19]. A Relay function is responsible for connecting AP entity with STA entity. A MAC protocol proposed by Kumar et al. [23] uses a static channel allocation mechanism for the distributed relay nodes. Enhancing this, a dynamic channel allocation and organization approach is proposed in [3] to improve the performance of relay-based large networks. Further to enhance relay to relay communication, Rao et al. [27] introduces dual-hop relay to extend the connectivity till the STAs. A cooperative relay with a cross-layer design for minimizing the power consumption of an IEEE 802.11ah network is proposed by Argyriou et al. [6]. However, the detailed deployments in different geographic locations are not considered while placing a relay node. None of these schemes consider elastic and inelastic heterogeneous traffic coexisting in the same IEEE 802.11ah network while utilizing the bandwidth efficiently. A Cognitive Radio (CR)based MAC protocol is proposed in [29], which regroups STAs considering the probability of collision. Nabuuma et al. [24] enhances the grouping scheme using a backoffbased approach with AID to reduce collisions in a sectorized network. Ahmadi et al. [15] A Received Signal Strength (RSS)-based solution to the hidden node problem. A STA senses the neighbors with the help of RSS values and ensures that nodes in the same group are known. However, which is not always true in dynamic conditions.

The efficiency of the RAW mechanism under nonideal channel condition is measured in [4]. Ali et al. [5] proposes an analytical model for differentiated QoS mechanisms of RAW, which is suitable in a heterogeneous IoT environment. A data-rate aware grouping scheme is presented in [28] which provides fair resource allocation in a network. Moreover, dynamic bandwidth allocation at RAPs has been a well-established problem. Hussain et al. [18] identified the congestion problem in a relay-based multi-hop WiFi-based long-distance networks. A dynamic bandwidth allocation scheme was proposed to mitigate that problem. Cooperative relaybased approaches have been used for reliable and efficient transmission [17]. Considering the heterogeneous traffic demands of STAs, scalability support in RAPs and AP node is extremely important in large scale networks. In a RAP-based mechanism proposed in [2], the authors find a smaller size time frame to reduce endto-end delay and increase throughput in a multi-hop network. However, the funneling effect at AP and congestion at relay still exist in the network.

Synthesis: The current research works attempt to improve network scalability in many ways. However, the possibility of high collisions at the time of slot assignment with the dense network still persists. The centralized single-channel access mechanism in IEEE 802.11ah affects the network performance with many devices trying to access the channel during a time slot simultaneously. Moreover, a hierarchical and multi-hop network faces challenges such as high latency and jitter. Further works are required to deal with low scalability, heterogeneity, and dynamicity issues in large-scale IoT.

\section{Table 1: List of Symbols}

\begin{tabular}{|c|l|}
\hline Symbol & Semantics \\
\hline$P_{r}$ & Received signal strength \\
$G_{r}$ & Receiver antenna gain \\
$X_{\sigma}$ & Shadowing effect with standard deviation $\sigma$ \\
$L(d)$ & Propagation loss for distance $d$ \\
$L_{t}$ & Traffic load at relay at time $t$ \\
$T_{a s s o c}$ & Average association time \\
$T_{g_{i}}$ & Total association time for group $g_{i}$ \\
$N_{n}^{k}$ & Number of RAPs required at $\mathrm{k}^{t h}$ hop \\
$T h_{t x o p}^{\lambda}$ & Average throughput with the use of $\lambda$ channels \\
$b_{i, j}$ & Steady state probability from state i to j \\
$B_{\gamma}$ & Available bandwidth during $\gamma$ duration \\
$\tau$ & Successful transmission probability of a STA in \\
& a contention slot \\
\hline
\end{tabular}

\section{The Proposed Protocol}

The design of the proposed protocol is greatly influenced by the multiple available 1 and $2 \mathrm{MHz}$ bandwidth channels in sub-1 GHz and flexible MCSs proposed in IEEE 802.11ah. The available channels are distributed among the nodes spread over various sectors in a larger coverage area. It allows simultaneous channel access in different RAW groups implemented by the sectors. A TXOP-based mechanism is also used by the RAP and AP nodes for faster data sharing. 


\subsection{Network Topology}

The RAP nodes are be connected with the AP using multi-hop distances. Figure 1 shows a typical IoT network architecture using IEEE 802.11ah where three IoT networks are connected to the Internet.

The architecture can be thought of as a combination of sensor and backhaul networks. The overall network can be represented as a set $N(A, R, E)=\{B(A, R), T(R, E)\}$, where $B$ and $T$ are the backhaul and sensor networks respectively. Here, $A$ is the AP node, $R$ is the set of RAP nodes $\left(R=R_{1}, R_{2}, \ldots, R_{n}\right)$, and $E$ is the set of end nodes (sensor/actuator) $\left(E=E_{1}, E_{2}, \ldots, E_{m}\right) . A$ takes the responsibility of initialization, synchronization, slot assignment, and channel allocation of the whole network. The $R$ s are the owners of channels and hold the responsibility of forwarding data from $E$ s to $A$ and vice versa.

\subsubsection{Relay Organization}

The RAP nodes are as powerful as the AP node. An RAP node carries out operations like association, RAP organization, and synchronization. An organization and planning scheme is important to use as less number of RAPs as possible. We can calculate the coverage range of AP or RAP nodes by using the signal strength of the radio. The IEEE 802.11 ah standard uses the macro model of propagation [16] for $\mathrm{Tx} / \mathrm{Rx}$. Considering an antenna height of 15 meters above a rooftop, the propagation loss (in $\mathrm{dB}$ ) in this model can be calculated as:

$L(d)=8+37.6 \log _{10}(d)+21 \log _{10}\left(\frac{f}{900 M H z}\right)$

where $d$ is the wireless distance between the sender and the receiver node, and $f$ is the carrier frequency. The value of $d$ can be calculated as, $d=10^{\left(T_{X P}-T_{R S S I}\right) / 20}$ [14], where $T_{R S S I}$ is the Received Signal Strength Indicator (RSSI). It depends on the fading, carrier frequency, loss, and sensitivity. $T_{X P}$ is the transmission power of an IEEE 802.11ah node. An edge node (at distance $d$ ) switch its working mode to relay for multihop overage. Enhancing [3], another RAP node is selected using angular separation (refer Figure 2), $\Delta \theta=$ $\frac{360^{\circ} \times 2 D}{2 \pi d}$, where $D$ is the RAP node coverage. As the coverage of RAP and AP are the same, the value of $d$ and $\mathrm{D}$ are also same, hence $\Delta \theta=\frac{360^{\circ}}{\pi}$. So, the required number of RAPs in 1-hop distance can be calculated as, $N_{n}^{1 \text { hop }}=\left\lceil\frac{360^{\circ}}{\Delta \theta}\right\rceil$. Similarly, angular separation for $k$-hop RAP node is given by $\Delta \theta / k$. We can calculate the number of RAP nodes in $k^{t h}$ hop as:

$N_{n}^{k}=\left\lceil\frac{k \times 360^{0}}{\Delta \theta}\right\rceil$
This procedure gives the lowest number of RAPs to be deployed around the AP node. Once the nodes are organized, the AP or RAP nodes can start association with the STAs.

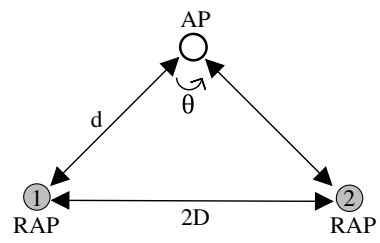

Fig. 2: Positioning of AP and RAP nodes in a network

\subsection{Node Association}

To solve the problem of a huge delay in association procedure, IEEE 802.11ah has proposed Centralized Authentication Control (CAC), as well as Distributed Authentication Control (DAC) [8]. We use CAC for associating STAs, which are distributed over different RAPs. The fast association mechanism is initiated by the AP or an RAP node which transmits an authentication control threshold $(\theta)$ value along with the beacon. A STA selects a random value $(\vartheta)$ within the window range of $[0,1022]$ and then associates itself with $A$ or $R$ only if $\vartheta<\theta$. Figure 3 shows an example scenario of the distributed association mechanism. Total number of association groups is $n+1$ ( $n$ number of distributed active $R \mathrm{~s}+A$ 's group $\left.\left(g_{a}\right)\right)$. Hence, the total association time can be calculated as:

$T_{\text {assoc }}=\max \left(T_{g_{a}}, T_{g_{i}}\right), \forall i=1,2, \ldots, n$

where $T_{g_{a}}$ and $T_{g_{i}}$ are the association time required for STAs to get associated with the nearest AP and RAP respectively.

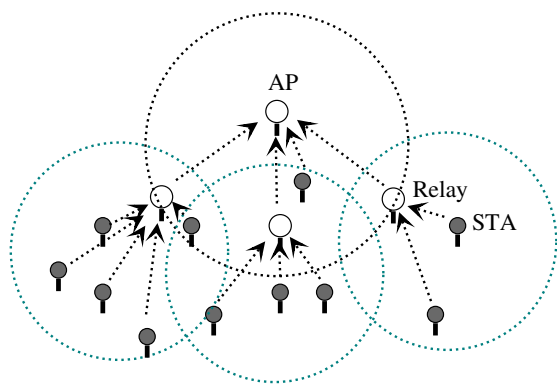

Fig. 3: Example scenario of node association 


\subsubsection{A Multi-band Sectorization Scheme}

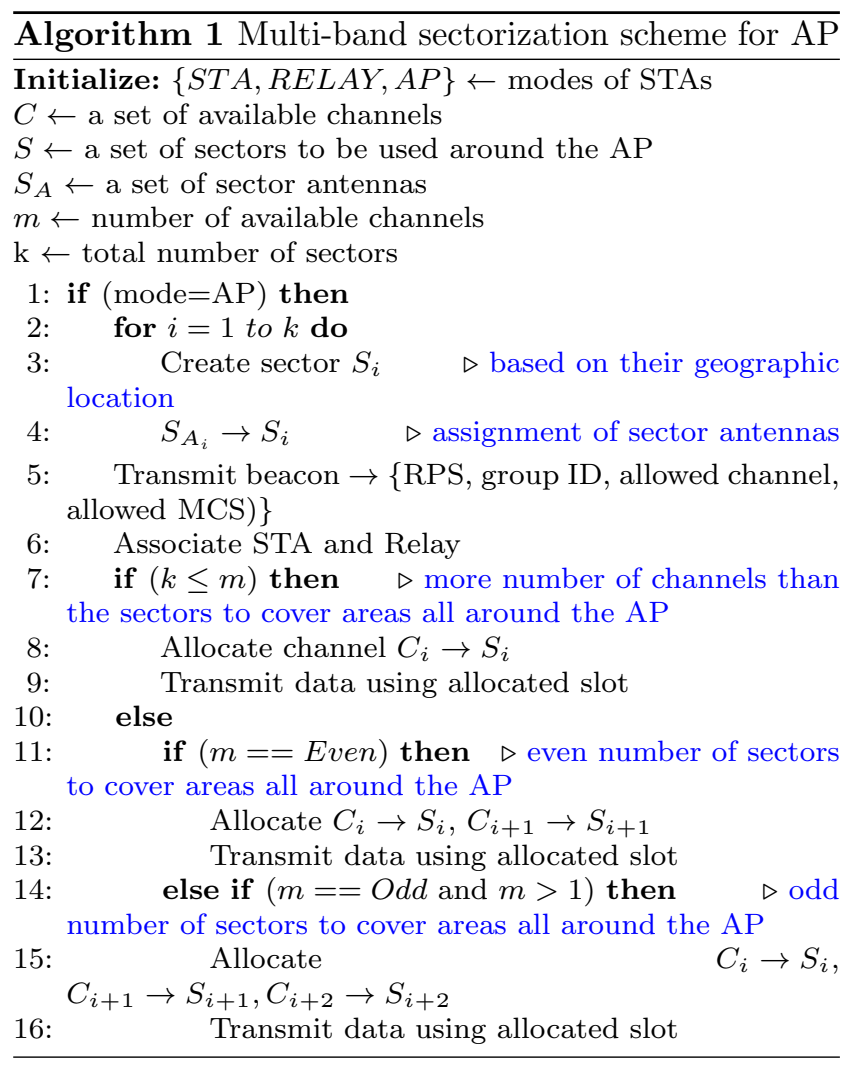

IEEE 802.11ah proposes a sectorization scheme to partition the coverage of a BSS into different non-overlapping sectors. The AP uses beacon to inform the transmission slots for different sectors. The beacon carries sector option elements with the group ID. Each STA is allocated a group ID to enable transmission in different sectors around the AP using Time Division Multiplexing (TDM). With the support of the proposed RAP solution and multiple available channels, we have incorporated two features - (i) multi-band sectorized transmission using Frequency Division Multiplexing (FDM), and (ii) configurable RAPs with multiple channels and MCSs, to the group sectorization scheme.

The current sectorization scheme proposed for IEEE 802.11ah dominantly uses TDM-based approaches. These schemes solve hidden node problems up to a certain extent without any improvement in overall network capacity. The proposed solution allows the AP node to use multiple channels and hence STAs from different sectors can transmit simultaneously using different channels. The number of radios employed in an AP depends on the number of available channels. For $N$ number of channels, the same number of radios and sector antennas are required. Due to the limited number of available channels, we restrict the value of $N$ to 4 . In this case, the angular beam of a sector antenna is $360 / N$ degree. Here, we consider four sectors to cover all around the AP (can be seen in Figure 4). Algorithm 1 discusses the proposed sectorization scheme. Lines \#1-4 allocates the sector antennas to the radios that are mounted in an AP. Lines \#7-15 discusses the channel allocation and transmission procedure. STAs or RAPs check the received signal strength $\left(P_{r}\right)$ from the AP through one or more sectorized beacons and join a better sector. Considering the fast fading effect, the power received at the receiver $\left(P_{r}\right)$ is given by [24]:

$P_{r}=P_{t}+G_{t}+G_{r}-\left(L(d)+X_{\sigma}\right)$

where $P_{t}$ is the received power, $G_{t}$, and $G_{r}$ are the transmitter and receiver gains respectively. With a zeromean log random variable and standard deviation $(\sigma)$, $X_{\sigma}$ gives the shadowing. The STAs or RAPs collect other information like group ID, RPS, and channel information from the beacon.

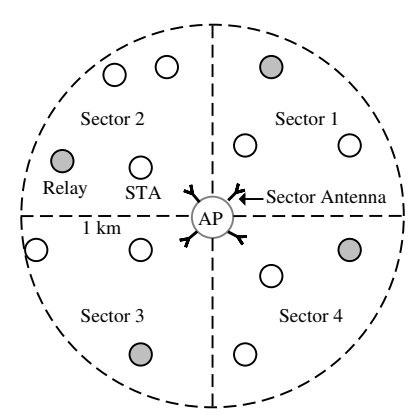

Fig. 4: Sector configuration around the AP node

\subsection{Dynamic Bandwidth Adjustment}

The proposed protocol enables the STAs to use different MCSs according to their data-rate requirements. The RAP nodes check the current load of itself in a periodic manner. The load is proportional to the amount of time wasted in collisions for a time duration $(t)$ [13], which can be calculated as:

$$
L_{t}=\frac{T_{c o l}}{T_{c o l}+T_{i d l}+T_{b u s}}
$$

where $T_{c o l}, T_{i d l}$, and $T_{b u s}$ are the collision, idle, and busy times respectively. The value of $L_{t}$ lies between 0 and 1 ; lower bound 0 says that there is no collision and upper bound 1 means there are collisions in the complete $t$ duration. Considering application's traffic requirements, number of STAs, and network conditions, 


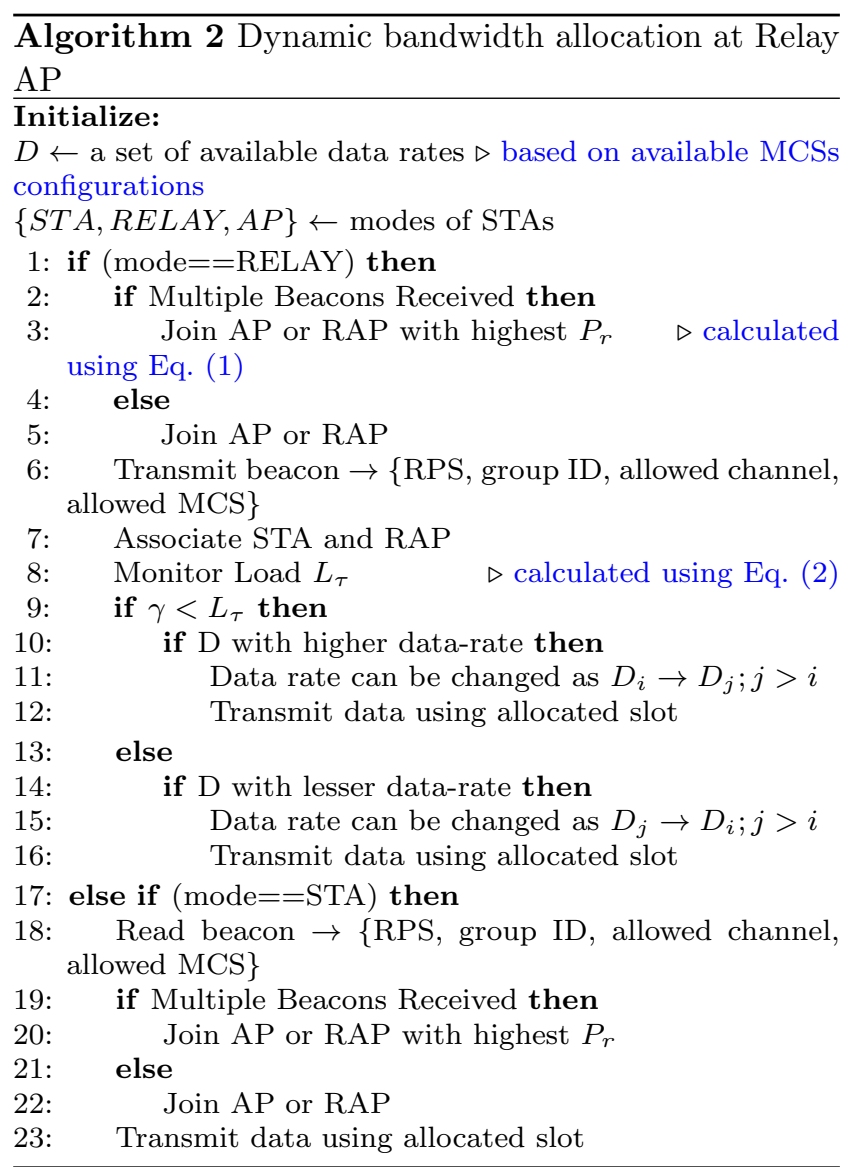

a factor $B_{\gamma}$ is chosen. $B_{\gamma}$ is the current available bandwidth of an RAP, calculated for a duration $\gamma$. The RAP chooses an MCS with higher data-rate when $L_{t}>B_{\gamma}$ and lower MCS for $L_{t}<B_{\gamma}$.

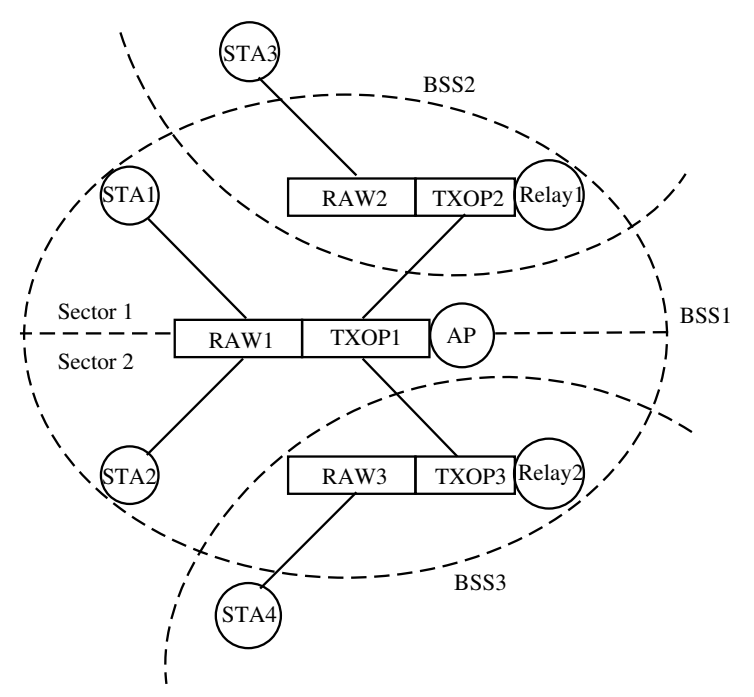

Fig. 5: Schematic representation of distributed RAW and TXOP

\subsection{Channel Access Mechanism}

In the proposed enhancement, we use a maximum of three different levels of RAWs. The AP and RAP nodes broadcast their RPS element with the beacon frame for the children $R$ s and $E$ s which are connected with AP directly. To communicate with STA and RAP or AP, RAW frame and TXOP frame are used respectively. For better efficiency, our solution dynamically use Block$A C K$ and $B D-T X O P$ for directional and bidirectional communication, respectively. If channels are sufficiently available, RAP further can use a different channel. But, to communicate with AP, the relay needs to switch to its parent AP or RAP's channel (refer Algorithm 2).

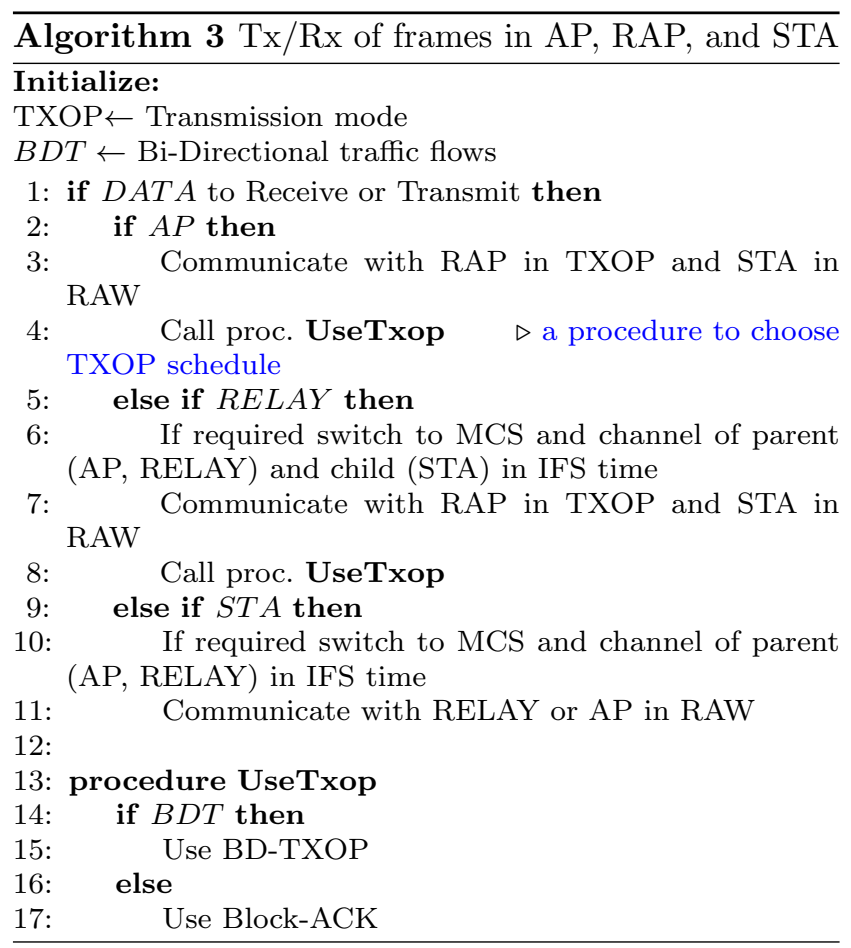

After selecting the RAW and TXOP, a node can initiate channel access. If a STA needs to transmit, it sends a PS-Poll frame using DCF as shown in Figure 6. Once successful, a MAC Protocol Data Unit (MPDU) can be transmitted. If AP needs to send a frame, it informs through the TIM beacon and then waits for a PS-Poll frame from the STA. AP to RAP or RAP to RAP access mechanism is done using BD-TXOP for better efficiency. As shown in Figure 6, in BD-TXOP an MPDU is sent without waiting for ACK. more data field of the header is used to inform the destination about pending data. The more data field is set to 1 or 0 to notify about more data or no more data pending respectively. The data $\mathrm{Tx} / \mathrm{Rx}$ procedure of the proposed scheme is discussed in Algorithm 3. 


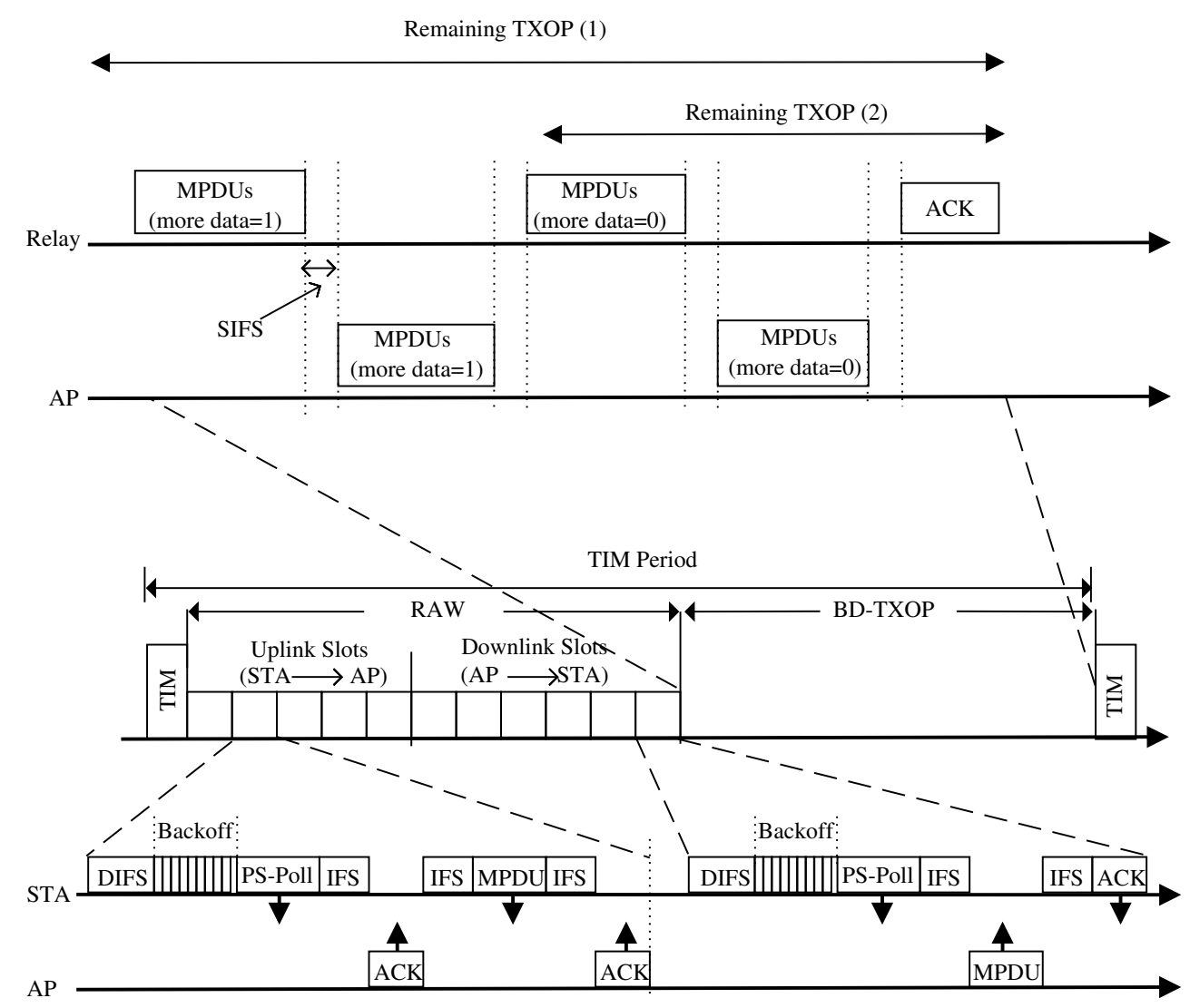

Fig. 6: RAW and TXOP based access in the proposed multi-hop network

\section{Performance Evaluation}

To validate the proposed scheme, initially, we carry out a theoretical analysis of throughput enhancement of the proposed scheme. After that, an extensive simulation analysis of the proposed, the traditional IEEE 802.11ah, and one of the most relevant state-of-the-art schemes are carried out and their results are systematically compared.

\subsection{Theoretical Analysis}

Bianchi et al. [11] defines saturation throughput (Th) as the average amount of payload bits transmitted successfully in a slot over the average duration of a slot time. The proposed protocol allows STAs to use noninterfering channels for simultaneous transmission. Therefore, contention happens with STAs belonging the same group and using the same channel. The state $(s(t), b(t))$ indicates that at time $t$, a node or the AP is at backoff stage $\mathrm{s}(\mathrm{t})$ with backoff counter $b(t)$. We assume the maximum backoff stages, $m$ ' and retry limit, $m$. When the value of the node's backoff counter becomes zero, it transmits. So, the transition probabilities can be given by,

$\left\{\begin{array}{lr}P\{i, j \mid i, j+1\}=1 & j \in\left[0, W_{i}-2\right], i \in[0, m] \\ P\{i, j \mid i-1,0\}=\frac{p}{W_{i}} & j \in\left[0, W_{i}-1\right], i \in[0, m] \\ P\{0, j \mid i, 0\}=\frac{1-p}{W_{0}} & j \in\left[0, W_{0}-1\right], i \in[0, m-1] \\ P\{0, j \mid m, 0\}=\frac{1}{W_{0}} & \end{array}\right\}$

Based on the above transition probabilities, the steady sate probability, $b_{i, j}$ can be calculated as:

$b_{i, j}=\lim _{t \rightarrow \infty} P\{s(t)=1, b(t)=j\}, i \in[0, m], j \in\left[0, W_{i}-1\right]$

Then, the transmission probability of a STA can be calculated as [11]:

$\tau=\sum_{i=1}^{m} b_{i, 0}=\sum_{i=0}^{m} p^{i} b_{0,0}=\frac{1-p^{m+1}}{1-p} b_{0,0}$

The final equation to calculate the saturation throughput, $T h$ is given as follows:

$T h=\frac{P_{t x} P_{\text {suc }} E[\text { Payload }]}{\left(1-P_{t x}\right) t_{s l o t}+P_{t x} P_{\text {suc }} T_{\text {suc }}+P_{\text {col }} T_{\text {col }}}$

where probability $P_{t x}=1-(1-\tau)^{n}$ indicates that there are $n$ number of STAs in the network and each of them 
get at least one transmission opportunity in a slot. $P_{s u c}=\frac{n \tau(1-\tau)^{n-1}}{P_{t x}}$ is the probability of transmission occurring on a slot is successful, $s_{t}$ is the average duration of a slot, and $P_{c o l}\left(=\left(1-P_{s u c}\right) P_{t x}\right)$ is the collision probability. $T_{\text {suc }}$ and $T_{c o l}$ are the busy times for successful transmission and collision respectively. For IEEE 802.11ah, $T_{\text {suc }}$ and $T_{\text {col }}$ can be calculated as below:

$T_{s u c}=T_{F H}+T_{P S-P O L L}+T_{D A T A}+T_{A C K}+T_{S I F S}$ $+T_{D I F S}+3 T_{P}$

$T_{\text {col }}=T_{F H}+T_{D A T A}+T_{D I F S}+T_{P}+T_{\text {Timeout }}$

where $T_{D A T A}, T_{S I F S}, T_{P}, T_{A C K}, T_{\text {timeout }}$ and $T_{D I F S}$ are the Data, SIFS, Propagation, ACK, ACK-Timeout, and DIFS duration respectively. The time for data and control frame for IEEE 802.11ah is calculated in [26]. Accordingly, Eqns. 3 and 4 find the DATA and ACK frame in our scheme, respectively [26]:

$T_{D A T A}=\left\lceil\frac{8 \times\left(L+m_{h}\right)}{\frac{R}{\alpha} \times \beta}\right\rceil \times T_{s y m}+T_{P H Y}$

$T_{A C K}=\left\lceil\frac{8 \times L_{c t r l}}{\beta}\right\rceil \times T_{s y m}+T_{P H Y}$

where $L, m_{h}, R, \alpha, \beta, T_{s y m}, L_{c t r l}$, and $T_{P H Y}$ are MAC payload size, header size, basic data rate, number of bits in one Orthogonal Frequency-Division Multiplexing (OFDM), symbol duration of OFDM, size of control frame, and PHY header size respectively. The values of different parameters are mentioned in Table 2. Theoretically, the saturation throughput of the proposed protocol is calculated by multiplying throughout with number of channels $(\lambda)$ :

$T h_{\text {prop }}=\lambda \times T h$

With the use of TXOP in the proposed protocol, the value of $T_{s u c}$ can be updated as:

$$
\begin{aligned}
T_{\text {suc }}^{\text {txop }}= & T_{P S-P O L L}+\delta T_{D A T A}+T_{A C K}+ \\
& (\delta+1)\left(T_{S I F S}+T_{D I F S}+T_{P}+T_{F H}\right)
\end{aligned}
$$

where $\delta$ is the number of data frames that we can send in a TXOP. Then, the saturation throughput will be:

$T h_{t x o p}=\frac{P_{t x} P_{\text {suc }}(\delta \times E[\text { Payload }])}{\left(1-P_{t x}\right) t_{\text {slot }}+P_{t x} P_{\text {suc }} T_{\text {suc }}^{t x o p}+P_{c o l} T_{c o l}}$

As a part of the proposed scheme, we can further implement multiple channels in AP. Then, the saturation throughput will be updated as:

$T h_{t x o p}^{\lambda}=\lambda \times T h_{t x o p}$

\subsection{Simulation Analysis}

The performance of the proposed enhanced and scalable MAC protocol (ES-MAC) is evaluated through extensive simulations using NS-3 [1]. Table 2 presents different parameters used in the simulation. Results are compared with Traditional access mechanism of IEEE 802.11ah and a sectorization scheme FE-MAC [10]. In FE-MAC, the AP node broadcasts beacons to the specific locations by utilizing the sectorized beams created with multiple antennas in it. We compare the throughput and delay performance of FE-MAC as shown in Figure 7a, 7b, and 7d. We analyze the proposed sectorization scheme in terms of throughout, delay and Packet Received Ratio (\%). Later, the overall network performance is measured considering different PRR enhancements.

Table 2: System and other parameters used in simula-

\begin{tabular}{|c|c|c|}
\hline Parameter & Value & \\
\hline Basic data rate & $650,300 \mathrm{Kbps}$ & \\
\hline Payload size & 256 Bytes & \\
\hline Radio propagation model & Outdoor (macro [7]) & \\
\hline OFDM symbol time $\left(T_{\text {sym }}\right)$ & $40 \mu s$ & \\
\hline MAC header & 14 bytes & \\
\hline PHY header & $6 \times T_{\text {sym }}$ & \\
\hline Slot time & $52 \mu s$ & \\
\hline SIFS & $16 \mu s$ & \\
\hline DIFS & SIFS $+2 \times$ Slot time & \\
\hline Modulation and Coding & MCS0, MCS1 & \\
\hline$\left[W_{0}, W_{m}\right]$ & {$[15,1023]$} & \\
\hline Backoff time & $\left(W_{0} / 2\right) \times$ Slot time & \\
\hline Initial backoff window & 64 & \\
\hline Types of traffic & UDP & \\
\hline Traffic interval & $700 \mathrm{~ms}$ & \\
\hline Simulation area & $2000 \mathrm{~m} \times 2000 \mathrm{~m}$ Flat-grid & \\
\hline Bandwidth & $1,2 \mathrm{MHz}$ & \\
\hline TXOP & $\begin{array}{l}\text { BlockACK } \\
\text { Directional }\end{array}$ & $\mathrm{Bi}-$ \\
\hline No. of STAs/RAPs (Max.) & $3000 / 100$ & \\
\hline RAW size & 15 & \\
\hline Group size & 8 & \\
\hline Beacon interval & $100 \mathrm{~ms}$ & \\
\hline
\end{tabular}
tion and theoretical analyses

\subsubsection{Association Time}

The RAPs create different Basic Service Sets (BSSs) in the network. Randomly scattered nodes get associated with the AP or RAP in a distributed manner. It is obvious that the association time of the proposed scheme is equal to the time required to associate all the STAs from the largest BSS. As shown in Figure 7, the total association time for 2000 devices in the proposed protocol is about 37 Seconds, whereas, in traditional IEEE 802.11ah, it is 42 Seconds. The RAP nodes are further supported with multiple MCSs, where it can choose an MCS with a higher or lower data-rate according to the current requirements of a $\mathrm{BSS}$. 


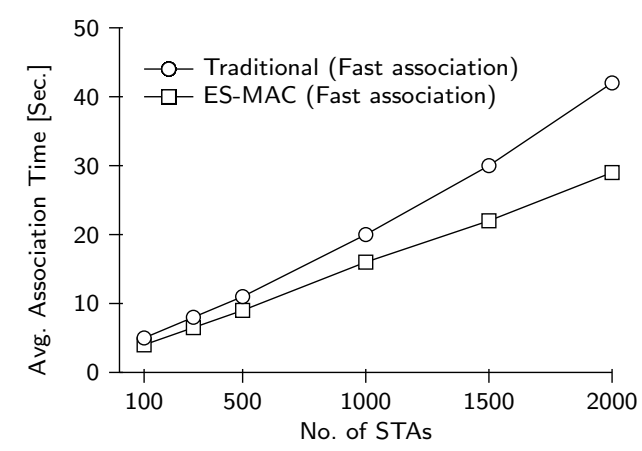

Fig. 7: Association time of the proposed scheme

\subsubsection{Throughput in Proposed Vs. Existing Schemes}

Figure 8 shows the throughput characteristics of the proposed scheme as compared to the traditional scheme with an increasing number of STAs. The throughput performance of the proposed scheme is significantly improved with 2, 3, and 4 sectors with the same number of channels. Although the use of multiple channels multiplies the available network data-rate capacity, due to the limited memory and processing capabilities of AP and RAP, the achieved throughput in the simulation seems to be lesser. In the case of $F E-M A C$ scheme with 2 sectors, the throughput performance is improved as compared to the traditional scheme. However, due to the lack of FDM support at AP and multi-channel operation, it gives lesser throughput than the proposed scheme. Due to the lack of multi-channel support in FE-MAC, the improvement with 2 sectors is sufficient to describe its efficiency. We compare the performance of the proposed and the traditional scheme considering 2 sectors over a varying number of RAW groups in a larger coverage area. We also analyze the proposed scheme with varying group sizes. In saturation conditions, more groups improve throughput performance as it reduces the collision domain. However, without a proper RAP organization over a larger coverage area, the hidden terminal problem remains a major concern. So, along with the RAW grouping for the STAs, our protocol uses a location-aware sectorized beacon from AP. The proposed scheme achieved superior performance as compared to the traditional one with an increasing number of groups (refer Figure 9).

To see the throughput performance over normal load conditions, we compare the proposed scheme considering a lesser number of STAs in the network. Traffic is generated with an interval of $0.5 \mathrm{Sec}$. As shown in Figure 10 , once the saturation point is reached, the performance of the proposed protocol starts deteriorating. The saturation point for the proposed scheme is higher than the existing schemes.

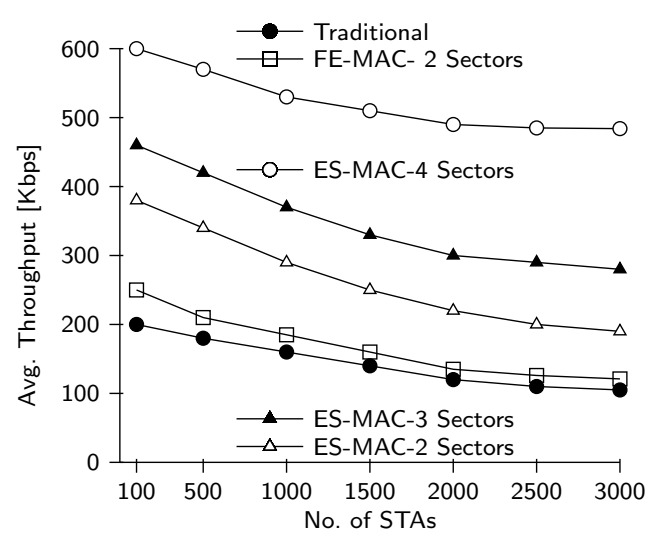

Fig. 8: Throughput performance at saturation load condition

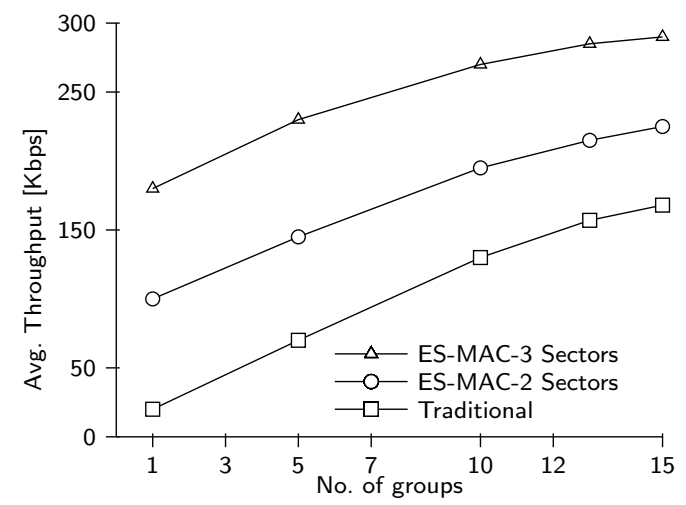

Fig. 9: Throughput performance with increasing number of groups

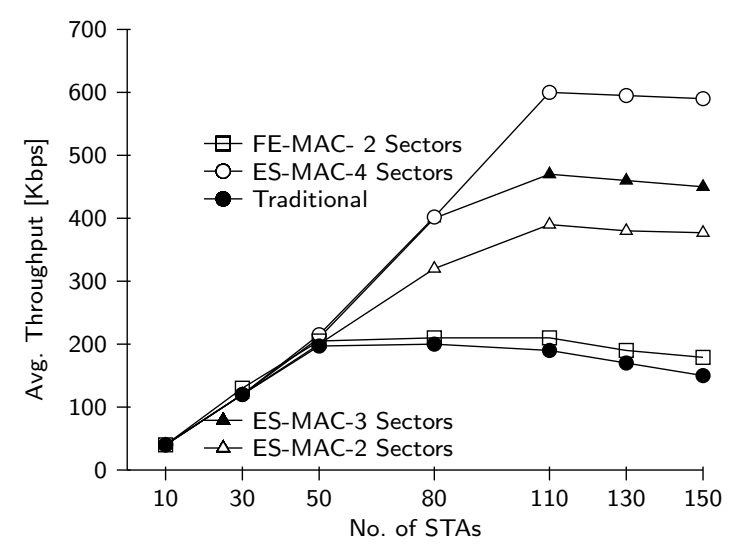

Fig. 10: Throughput performance at normal load scenario

\subsubsection{Delay in Proposed Vs. Existing Schemes}

As shown in Figure 11, the proposed scheme significantly improves the delay characteristics considering 2 to 4 sectors for the same number of channels. As dis- 


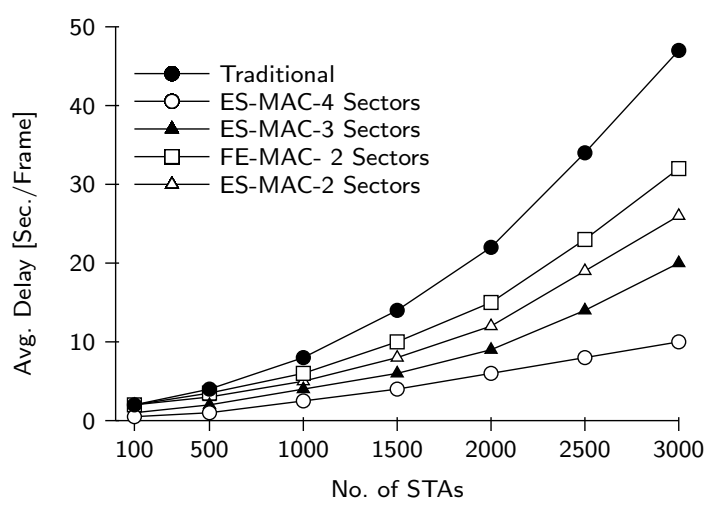

Fig. 11: Delay incurred with increasing number of stations

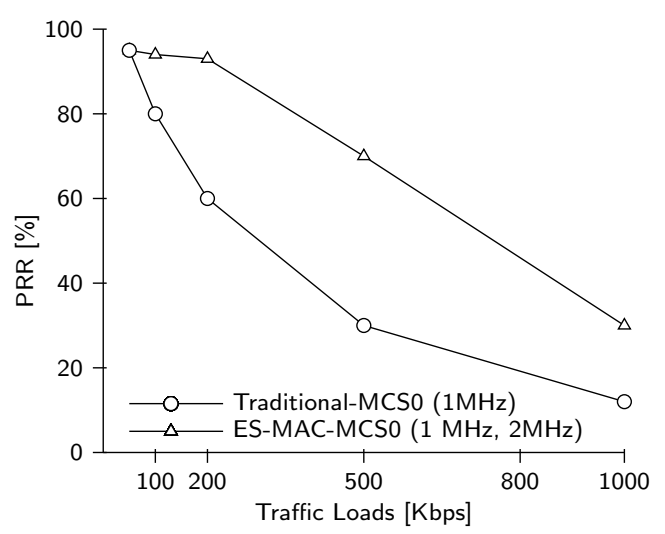

Fig. 12: Packet received ratio with dynamic load scenarios

cussed, the multiple channel operations supported in the scheme allows efficient transmission among different STAs in the network. The delay is reduced by the proposed protocol as shown in Figure 11. The proposed protocol allows simultaneous transmission in different sectors by using different channels. The RAPs added in a particular sector also transmit simultaneously as per the allocated channel. Further, when the number of STAs is divided based on the channels, the size of contention is also reduced, hence access delay is less.

\subsubsection{Dynamic Load Balancing}

The proposed scheme dynamically adjusts MCSs at the relay node for supporting varying load in the network, especially to facilitate the stations with their demands. IEEE 802.11ah has multiple MCSs with different channel configurations. In this analysis we consider, MCS0 with $1 \mathrm{MHz}$ and $2 \mathrm{MHz}$ bandwidth, which give $300 \mathrm{Kbps}$ and $650 \mathrm{Kbps}$ of data-rate, respectively. Varying traffic loads (100-1000Kbps) are generated from BSS to AP. With the use of MCS0 in the traditional 802.11ah-based

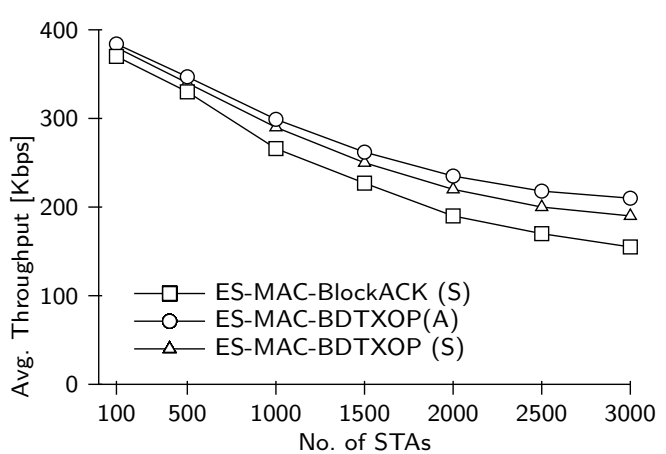

Fig. 13: Performance of the TXOP-based channel access mechanism

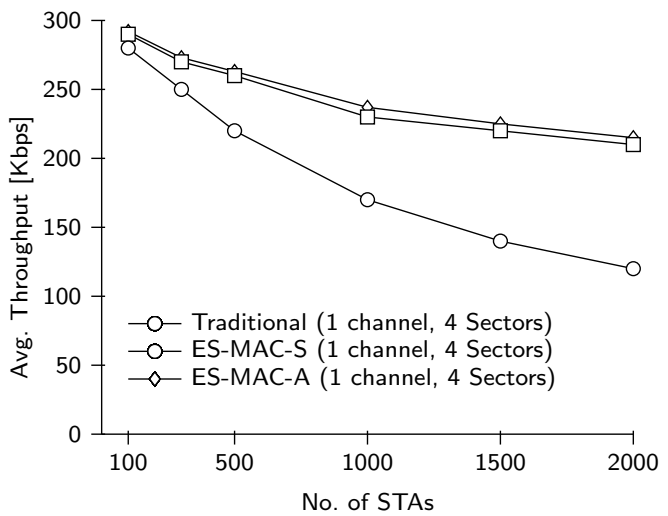

Fig. 14: Overall simulation and theoretical throughput performance

network, a maximum of $150 \mathrm{Kbps}$ throughput is possible. However, our solution provides up to $325 \mathrm{Kbps}$ throughput, which is due to the support of multiple MCSs. The RAPs in the proposed scheme switch their MCS to other MCSs having a higher data rate. Therefore, as shown in Figure 12, the proposed scheme performs almost $26 \%$ better $\operatorname{PRR}(\%)$ than the traditional one.

\subsubsection{TXOP-based Efficient Access for AP and RAP}

Finally, we have measured the efficiency of the BDTXOP scheme used in the proposed protocol compared to the BlockAck mechanism. As shown in Figure 13, a significant improvement can be seen in the BD-TXOPbased solution considering bi-directional traffic. As BDTXOP does not send ACK for all received packets, the overall efficiency is improved. 


\subsection{Comparison of Theoretical and Simulation Results}

The saturation throughput of the proposed protocol is calculated using the Markov Chain model. By using the Eqns. 5, 3, and 4, throughput characteristic is evaluated with increasing number of STAs. Figure 13 shows the trend of analytically computed results. Results of analytical (A) and simulation (S) studies are shown in Figure 13 and 14 use 1 and 2 channels respectively. The theoretical and simulation analyses display harmonized throughput performance in the same configuration. The proposed protocol exhibits $24 \%$ overall throughput improvement using single channel which is shown in Figure 14 .

\section{Conclusion}

This work presented a distributed, multi-sector, multichannel, and load-aware MAC protocol for IEEE 802.11ahbased large-scale networks. It shows significant performance improvement in throughput and delays over the traditional and state-of-the-art MAC schemes. The proposed distributed node association mechanism requires $11 \%$ lesser time than the traditional fast association scheme. Scalability issues at AP and RAP nodes are resolved by allowing simultaneous transmission using multiple channels and sectors. The dynamic load balancing and TXOP operation at the RAP node significantly increase the efficiency of large-scale IoT networks. In the future, we plan to optimize the time frame duration for AP and RAP nodes based on the heterogeneous traffic requirements of STAs.

\section{Conflict of Interest}

On behalf of all authors, the corresponding author states that there is no conflict of interest.

\section{References}

1. What is NS-3 (2020). Available at: https://www.nsnam.org/overview/what-is-ns-3

2. Ahmed, N., Misra, S.: Channel Access Mechanism for IEEE 802.11 ah-Based Relay Networks. In: ICC 20202020 IEEE International Conference on Communications (ICC), pp. 1-6. IEEE (2020)

3. Ahmed, N., Rahman, H., Hussain, M.I.: An IEEE 802.11ah-based scalable network architecture for Internet of Things. Annals of Telecommunications 73(7-8), 499-509 (2018)

4. Ali, M.Z., Mišić, J., Mišić, V.B.: Efficiency of restricted access window scheme of IEEE 802.11 ah under nonideal channel condition. In: International Conference on Green Computing and Communications, pp. 251-256. IEEE (2018)
5. Ali, M.Z., Mišić, J., Mišić, V.B.: Performance Evaluation of Heterogeneous IoT Nodes With Differentiated QoS in IEEE 802.11ah RAW Mechanism. IEEE Transactions on Vehicular Technology 68(4), 3905-3918 (2019)

6. Argyriou, A.: Power-efficient estimation in IEEE 802.11ah wireless sensor networks with a cooperative relay. In: International Conference on Communications (ICC), pp. 6755-6760. IEEE (2015)

7. Aust, S., Prasad, R.V., Niemegeers, I.G.: Outdoor longrange WLANs: A lesson for IEEE 802.11ah. IEEE Communications Surveys \& Tutorials 17(3), 1761-1775 (2015)

8. Bankov, D., Khorov, E., Lyakhov, A., Stepanova, E.: Fast centralized authentication in Wi-Fi HaLow networks. In: International Conference on Communications (ICC), pp. 1-6. IEEE (2017)

9. Banos, V., Afaqui, M.S., Lopez, E., Garcia, E.: Throughput and Range Characterization of IEEE 802.11 ah. IEEE Latin America Transactions 15(9), 1621-1628 (2017)

10. Bhandari, S., Sharma, S.K., Wang, X.: Device Grouping for Fast and Efficient Channel Access in IEEE 802.11ah based IoT Networks. In: IEEE International Conference on Communications Workshops (ICC Workshops), pp. 16. IEEE (2018)

11. Bianchi, G.: Performance analysis of the IEEE 802.11 distributed coordination function. IEEE Journal on selected areas in communications 18(3), 535-547 (2000)

12. Chen, C., Zhao, H., Qiu, T., Hu, M., Han, H., Ren, Z.: An efficient power saving polling scheme in the internet of energy. Journal of Network and Computer Applications (2017). Https://doi.org/10.1016/j.jnca.2017.01.002

13. Dhananjay, A.V.: Load estimation in IEEE 802.11 wireless networks (2008). Published at: Digital Repository Iowa State University, http://lib. dr. iastate. edu/

14. Dong, Q., Dargie, W.: Evaluation of the reliability of RSSI for indoor localization. In: International Conference on Wireless Communications in Underground and Confined Areas, pp. 1-6. IEEE (2012)

15. Ghasemiahmadi, M., Li, Y., Cai, L.: RSS-based grouping strategy for avoiding hidden terminals with GS-DCF MAC protocol. In: Wireless Communications and Networking Conference (WCNC), pp. 1-6. IEEE (2017)

16. Hazmi, A., Rinne, J., Valkama, M.: Feasibility study of IEEE 802.11ah radio technology for IoT and M2M use cases. In: IEEE GIOBECOM WORKSHOPS, pp. 16871692. IEEE (2012)

17. Hiraguri, T., Nishimori, K., Shitara, I., Mitsui, T., Shindo, T., Kimura, T., Matsuda, T., Yoshino, H.: A Cooperative Transmission Scheme in Drone-Based Networks. IEEE Transactions on Vehicular Technology 69(3), 2905-2914 (2020)

18. Hussain, I., Ahmed, Z.I., Saikia, D.K., Sarma, N.: A QoS-aware dynamic bandwidth allocation scheme for multi-hop WiFi-based long distance networks. EURASIP Journal on Wireless Communications and Networking 2015(1), 160 (2015)

19. IEEE: Wireless LAN Medium Access Control (MAC) and Physical Layer (PHY) Specifications: Amendment 2: Sub $1 \mathrm{GHz}$ License Exempt Operation. IEEE P802.11ah/D10.0, Sep 2016 pp. 1-660 (2016)

20. Kassab, W., Darabkh, K.A.: A-Z survey of Internet of Things: Architectures, protocols, applications, recent advances, future directions and recommendations. Journal of Network and Computer Applications p. 102663 (2020). Available at: https://doi.org/10.1016/j.jnca.2020.102663 
21. Khorov, E., Lyakhov, A., Krotov, A., Guschin, A.: A survey on IEEE 802.11ah: An enabling networking technology for smart cities. Computer Communications 58, 53$69(2015)$

22. Kocan, E., Domazetovic, B., Pejanovic-Djurisic, M.: Range extension in IEEE 802.11ah systems through relaying. Wireless Personal Communications 97(2), 18891910 (2017)

23. Kumar, S., Lim, H., Kim, H.: Hierarchical MAC protocol with Multi-channel allocation for enhancing IEEE 802.11ah Relay Networks. In: Wireless Communications and Mobile Computing Conference (IWCMC), pp. 14581463. IEEE (2015)

24. Nabuuma, H., Alsusa, E., Baidas, M.W.: AID-based backoff for throughput enhancement in 802.11ah networks. International Journal of Communication Systems 32(7), e3923 (2019)

25. Park, M.: IEEE 802.11ah: Energy efficient MAC protocols for long range wireless LAN. In: International conference on communications (ICC), pp. 2388-2393. IEEE (2014)

26. Raeesi, O., Pirskanen, J., Hazmi, A., Levanen, T., Valkama, M.: Performance evaluation of IEEE 802.11ah and its restricted access window mechanism. In: International Conference on Communications Workshops (ICC), pp. 460-466. IEEE (2014)

27. Rao, S.N., Akhil, P., Kumaravelu, V.B., Arthi, M.: Dual-Hop Relaying for Quality of Service Improvement in IEEE 802.11ah-Downlink. In: International Conference on Communication and Signal Processing (ICCSP), pp. 0249-0253. IEEE (2018)

28. Sangeetha, U., Babu, A.: Fair and efficient resource allocation in IEEE 802.11ah WLAN with heterogeneous data rates. Computer Communications 151, 154 - 164 (2020)

29. Shafiq, M., Ahmad, M., Irshad, A., Gohar, M., Usman, M., Khalil Afzal, M., Choi, J.G., Yu, H.: Multiple Access Control for Cognitive Radio-Based IEEE 802.11ah Networks. Sensors 18(7), 2043 (2018) 


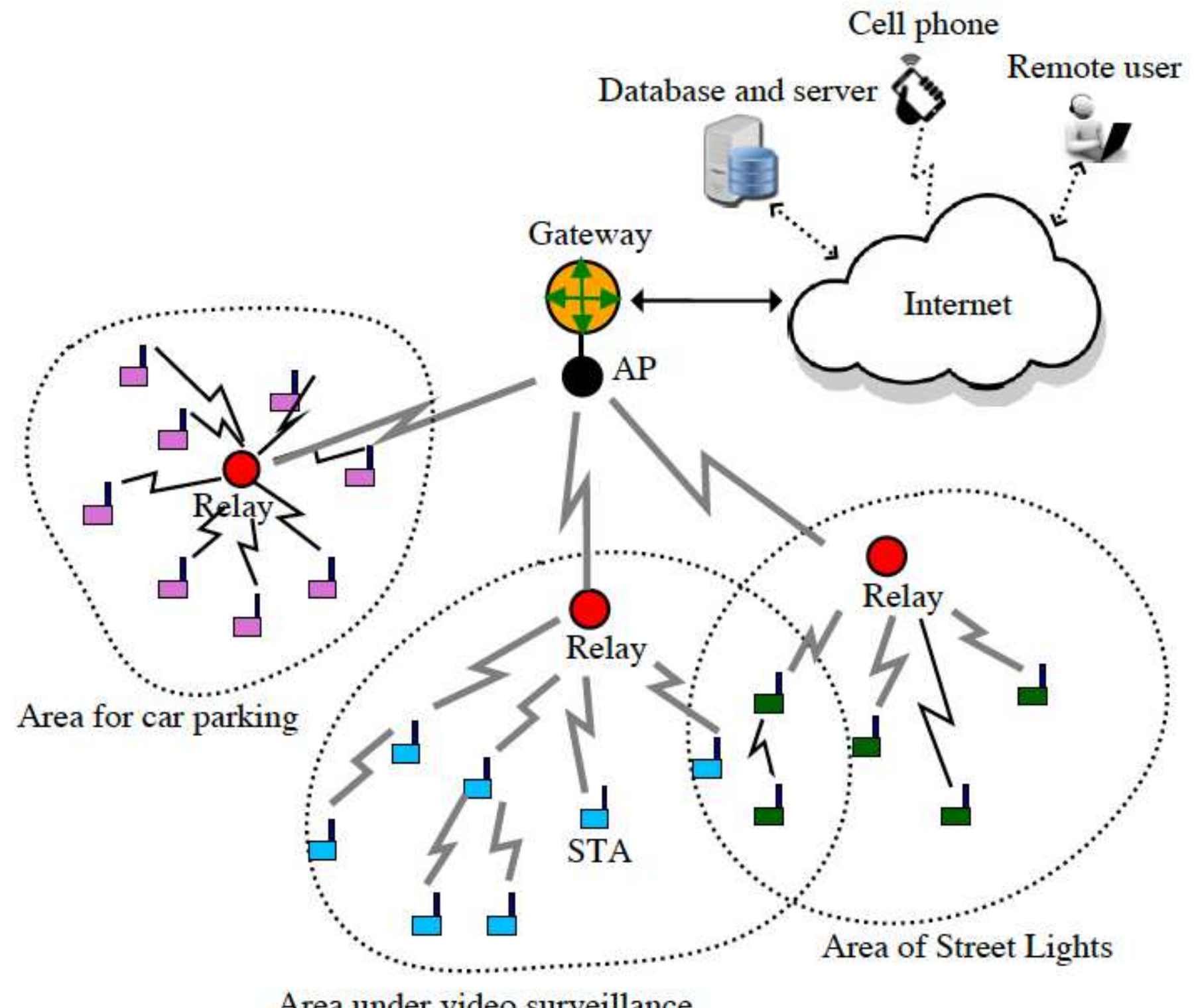

Figure 1

IEEE 802.11ah-based smart city network architecture spreading over large coverage area 


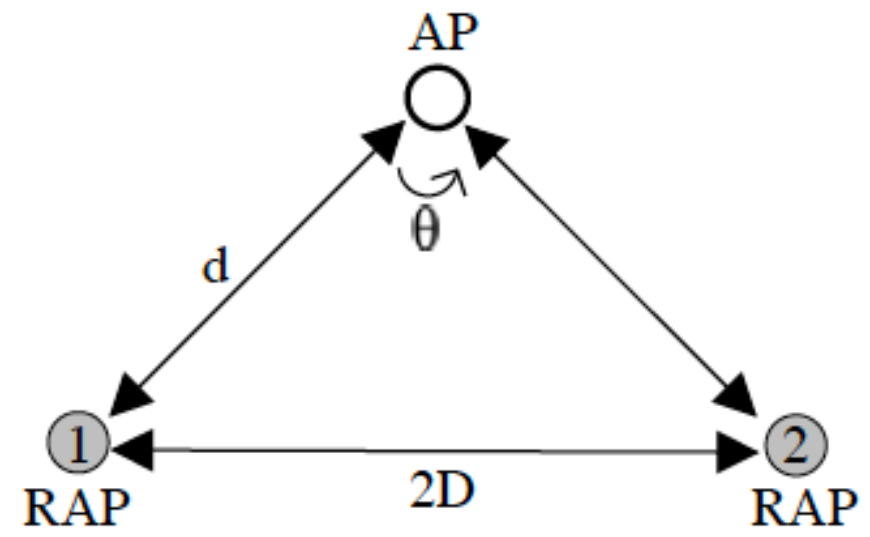

Figure 2

Positioning of AP and RAP nodes in a network

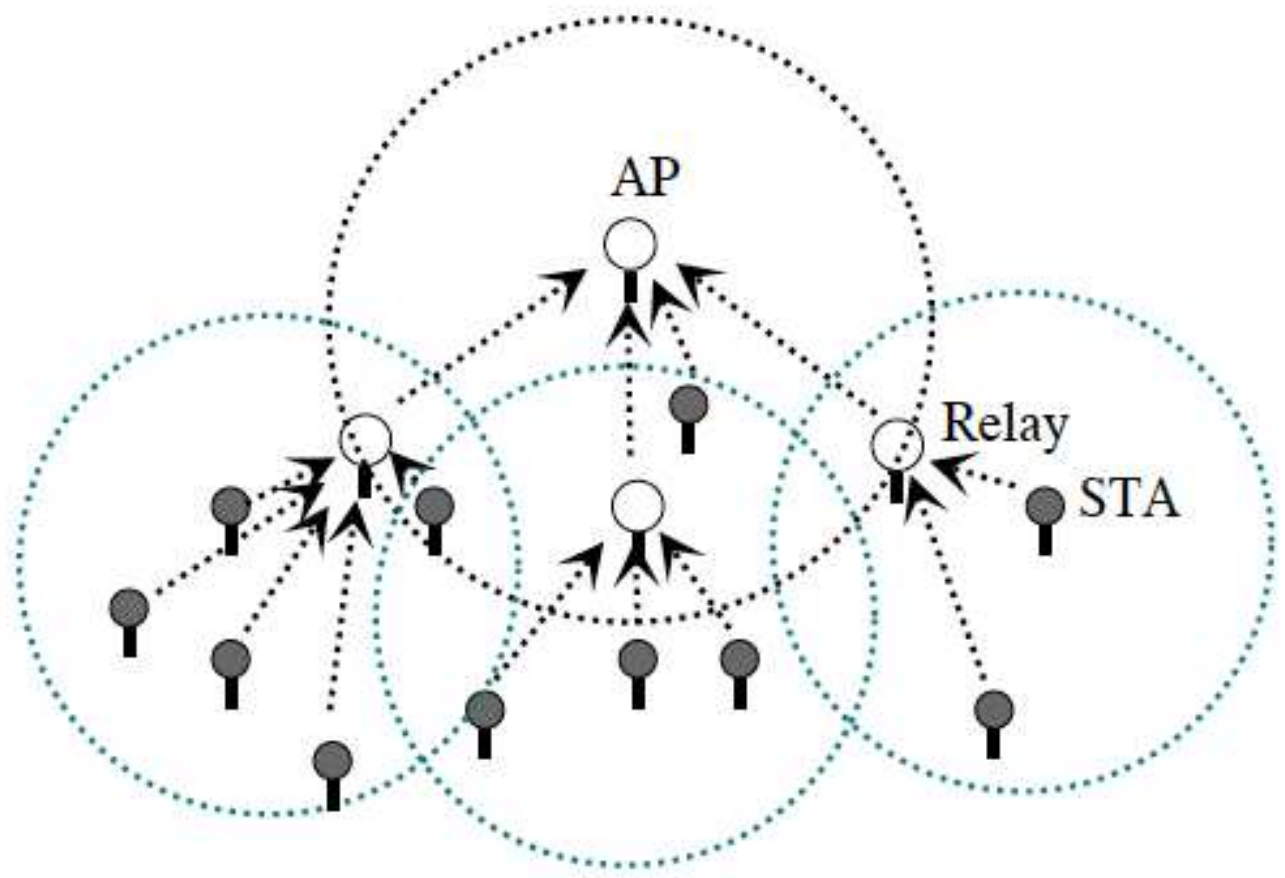

Figure 3

Example scenario of node association 


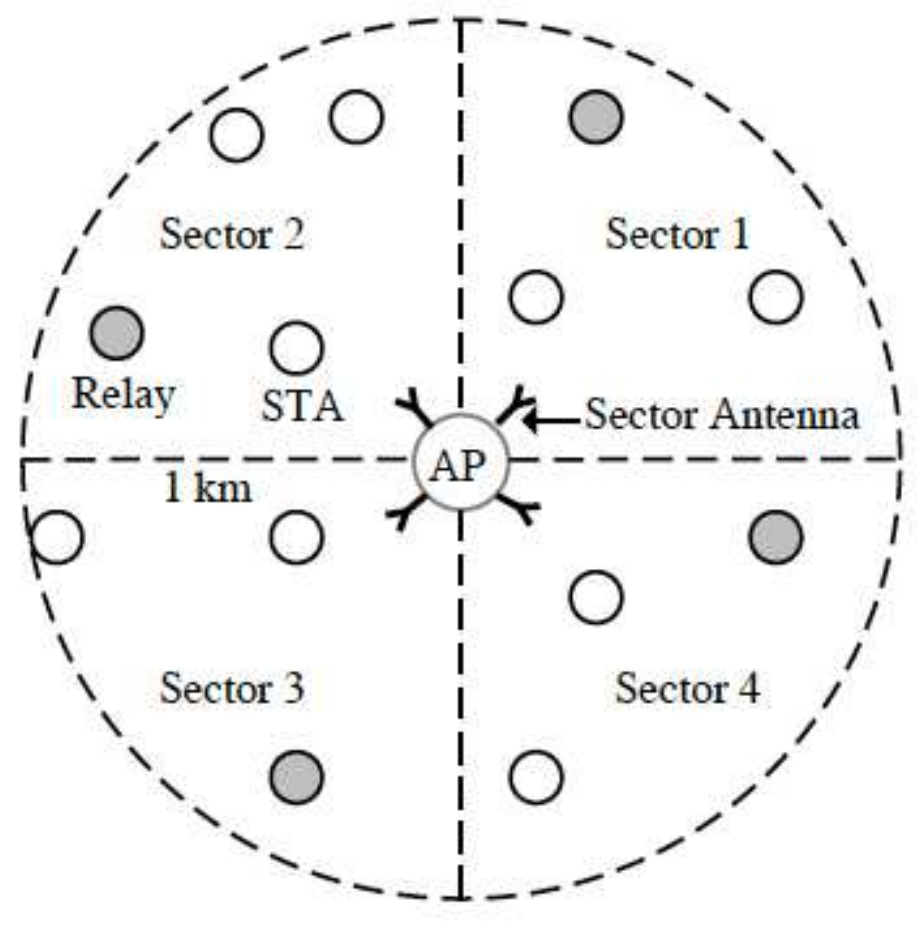

Figure 4

Sector configuration around the AP node 


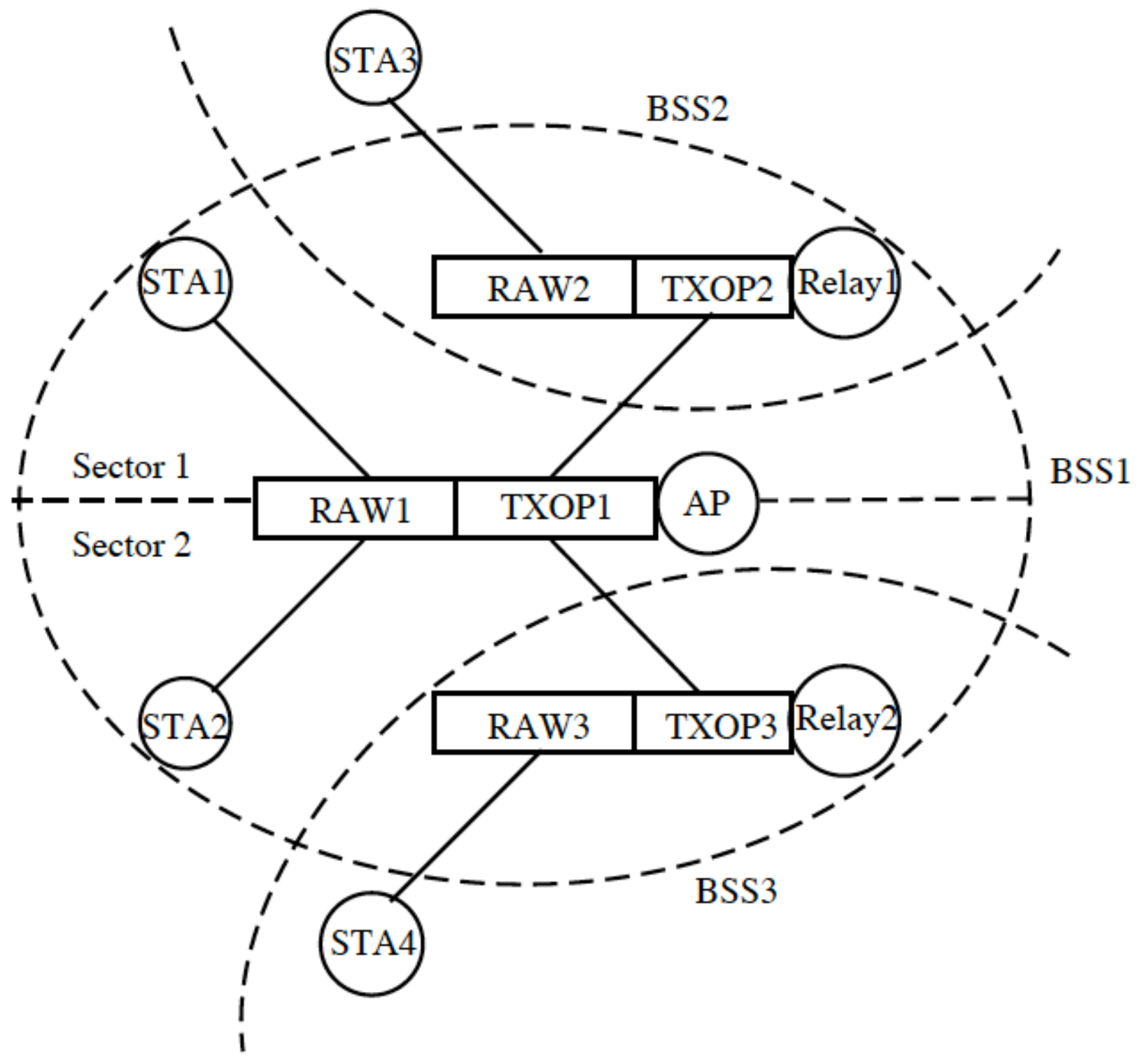

Figure 5

Schematic representation of distributed RAW and TXOP 


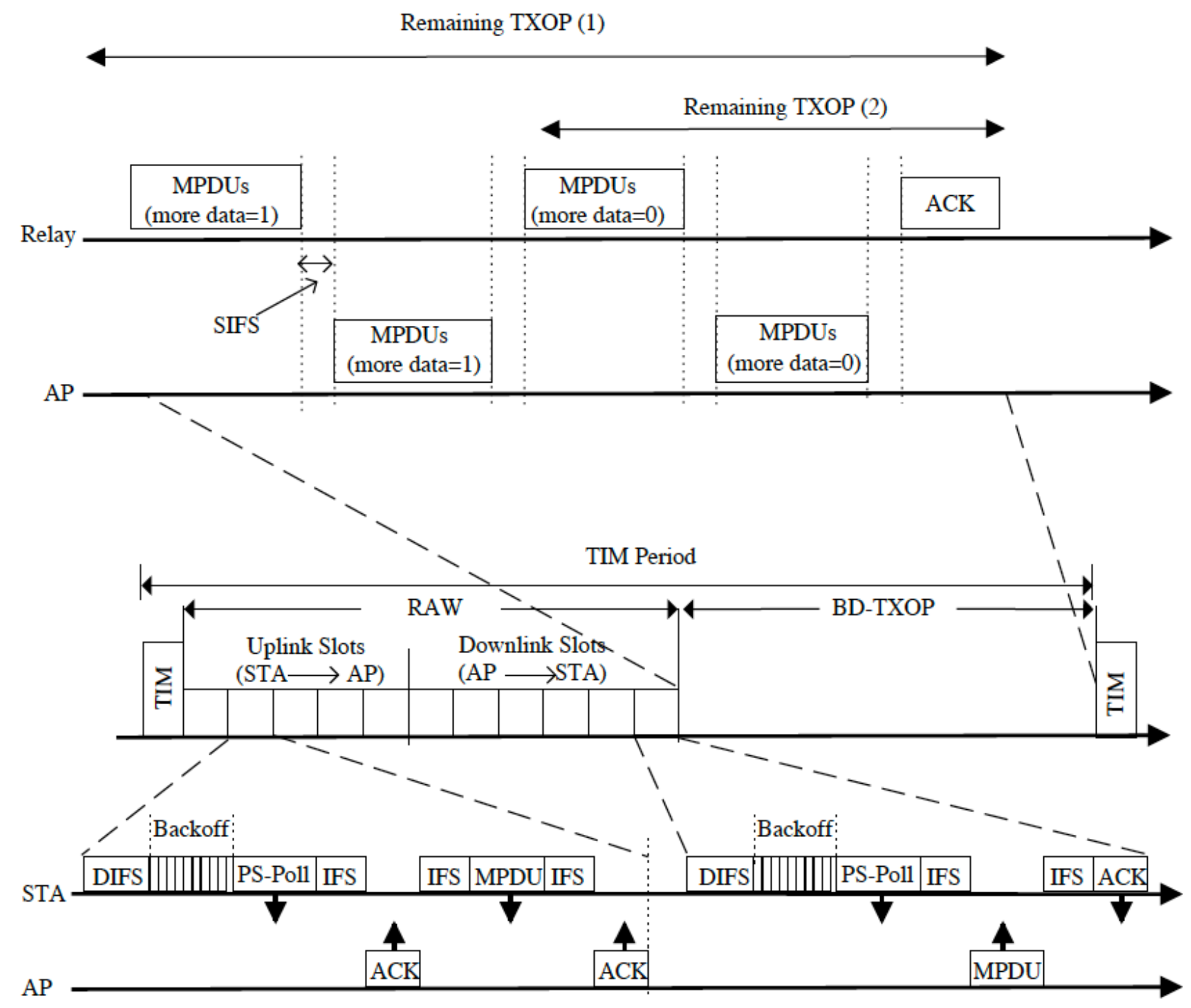

Figure 6

RAW and TXOP based access in the proposed multi-hop network 


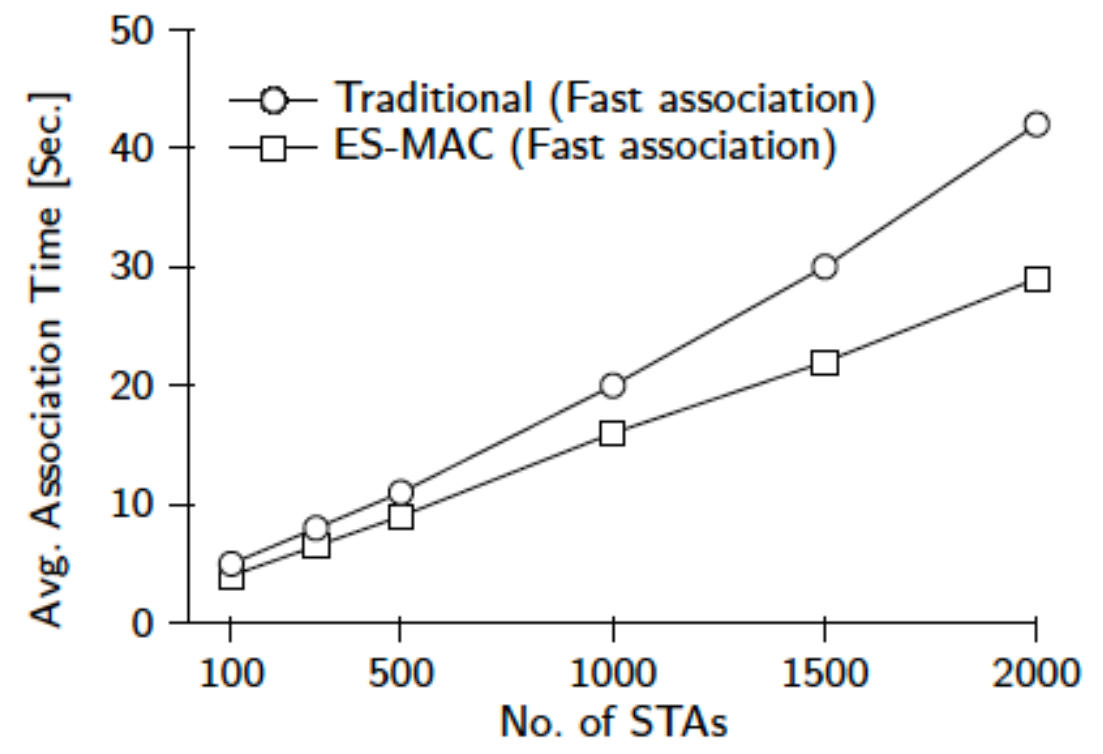

Figure 7

Association time of the proposed scheme

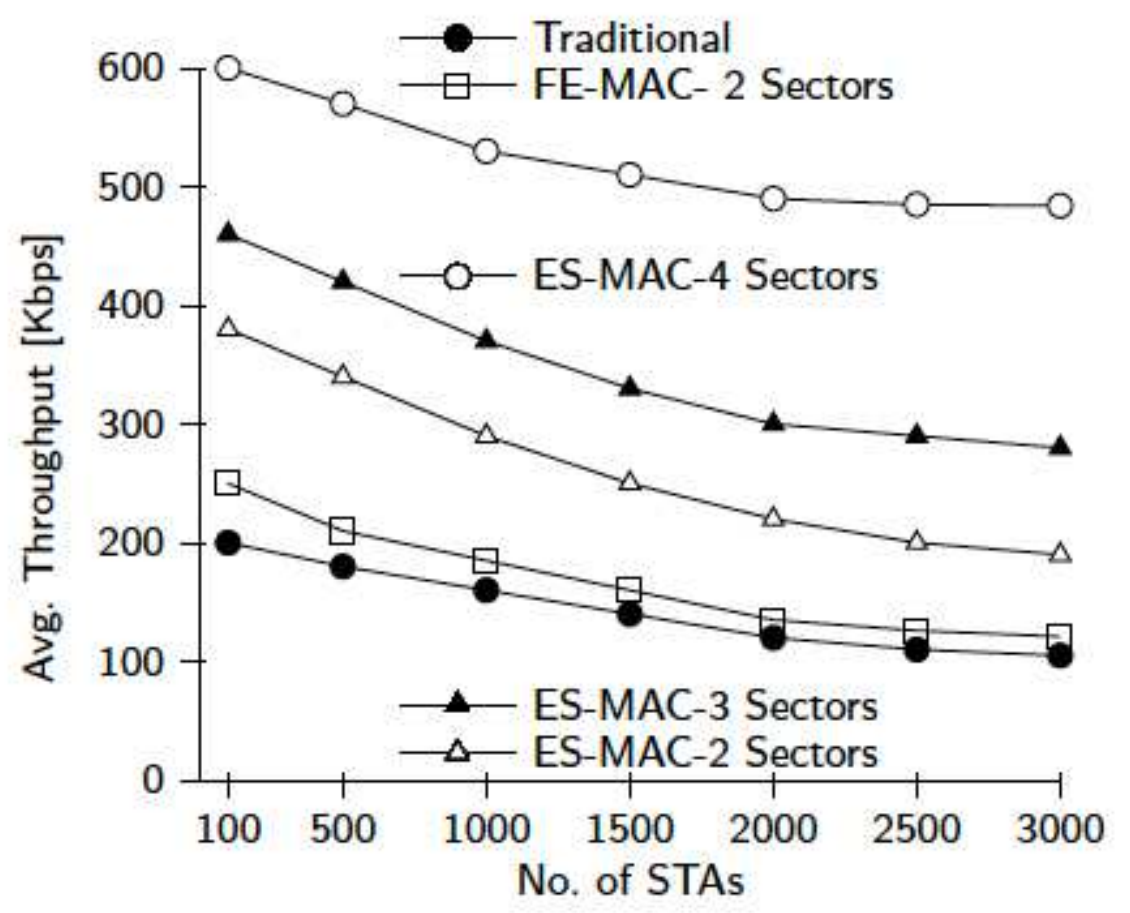

Figure 8

Throughput performance at saturation load condition 


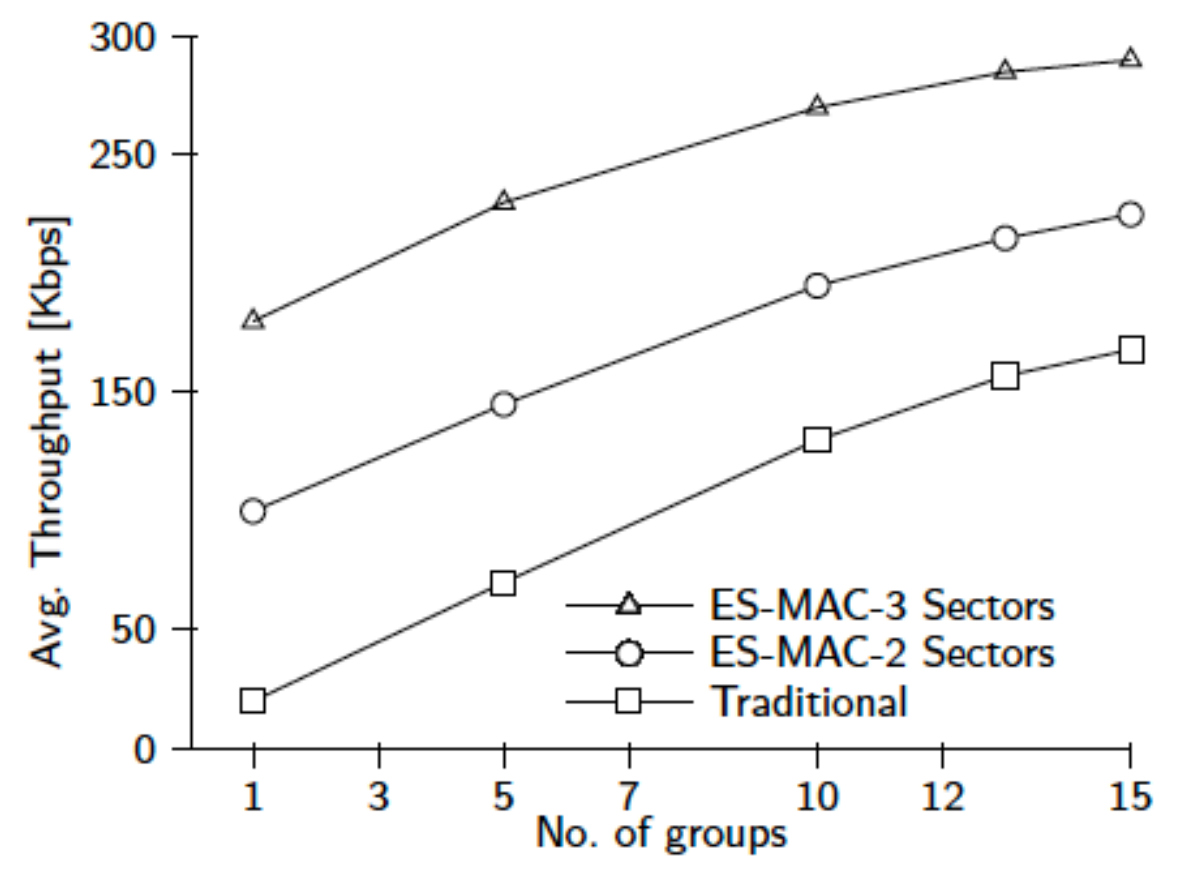

Figure 9

Throughput performance with increasing number of groups

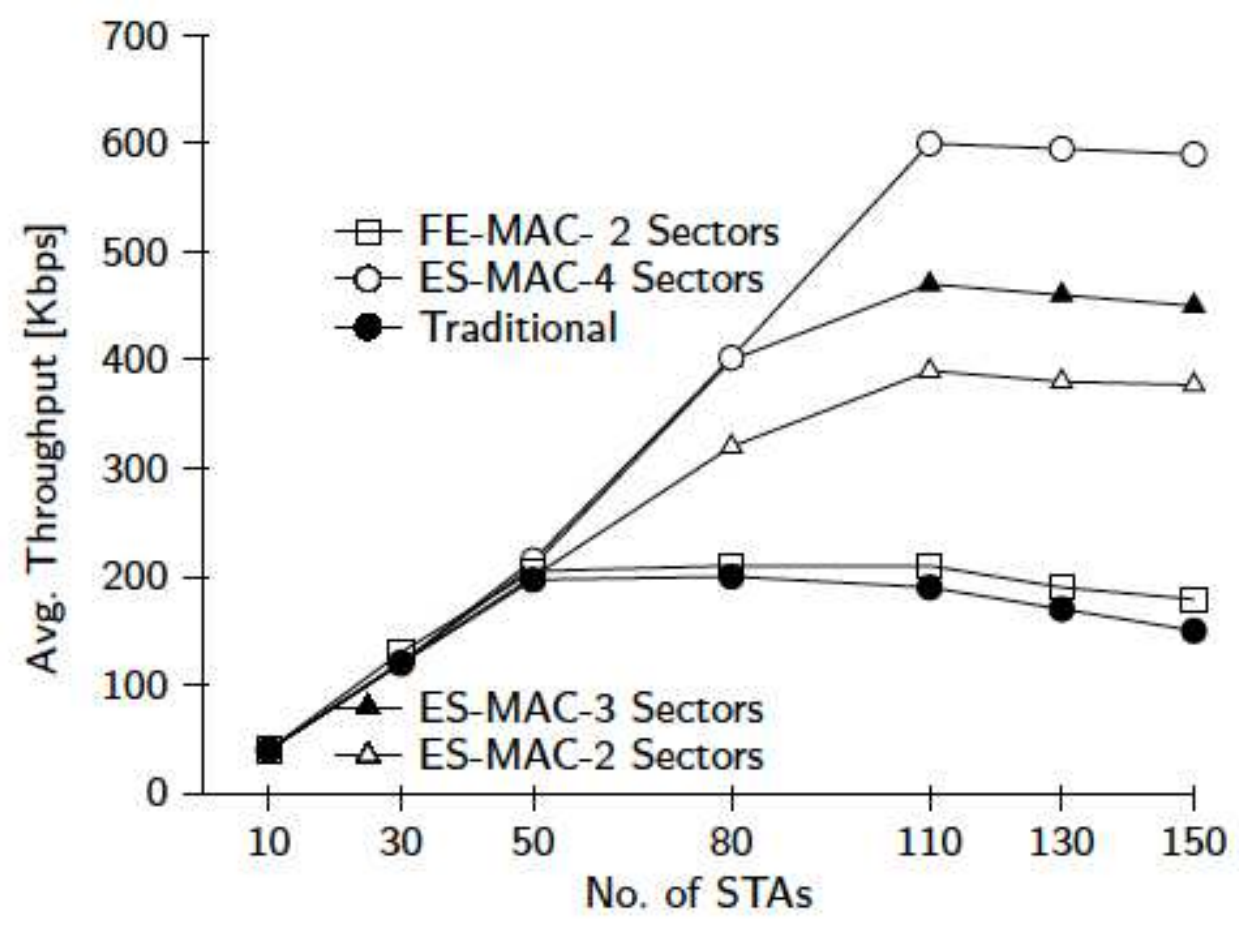

Figure 10

Throughput performance at normal load scenario 


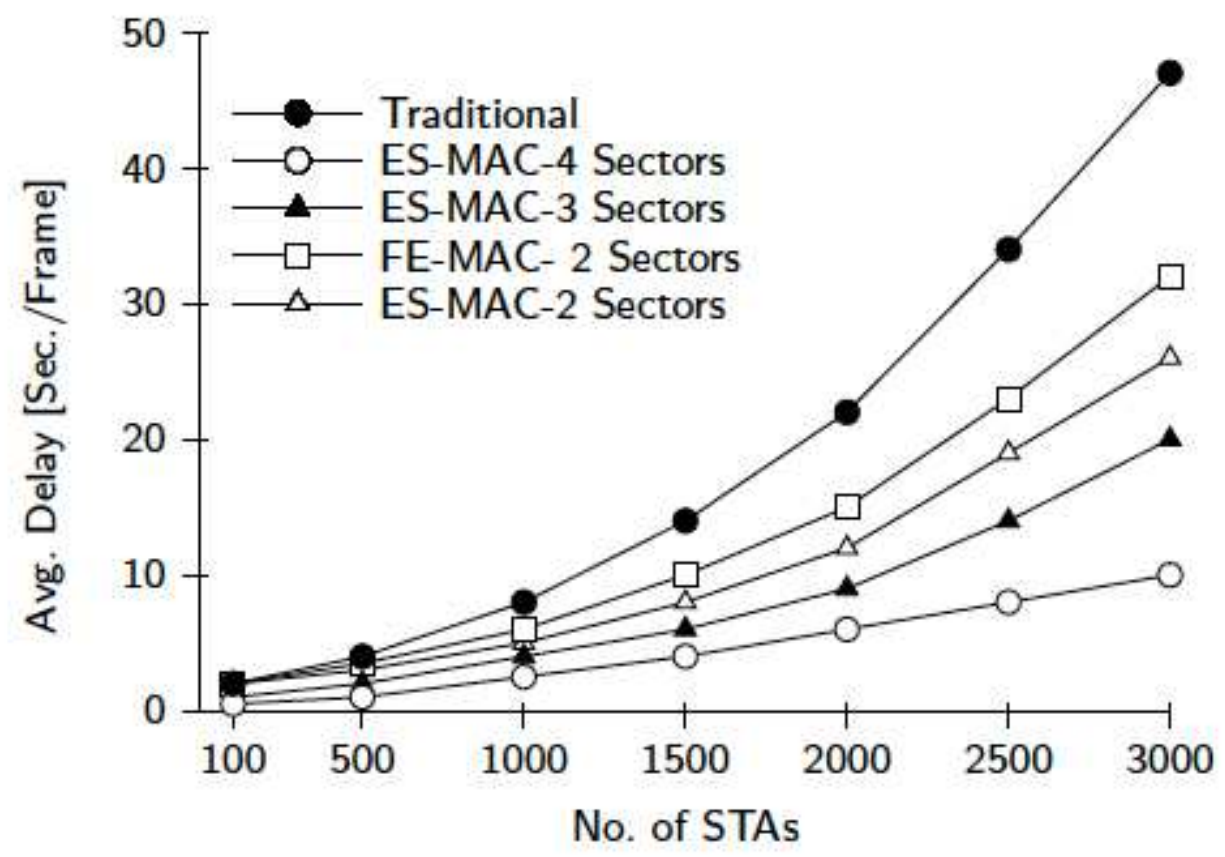

Figure 11

Delay incurred with increasing number of stations

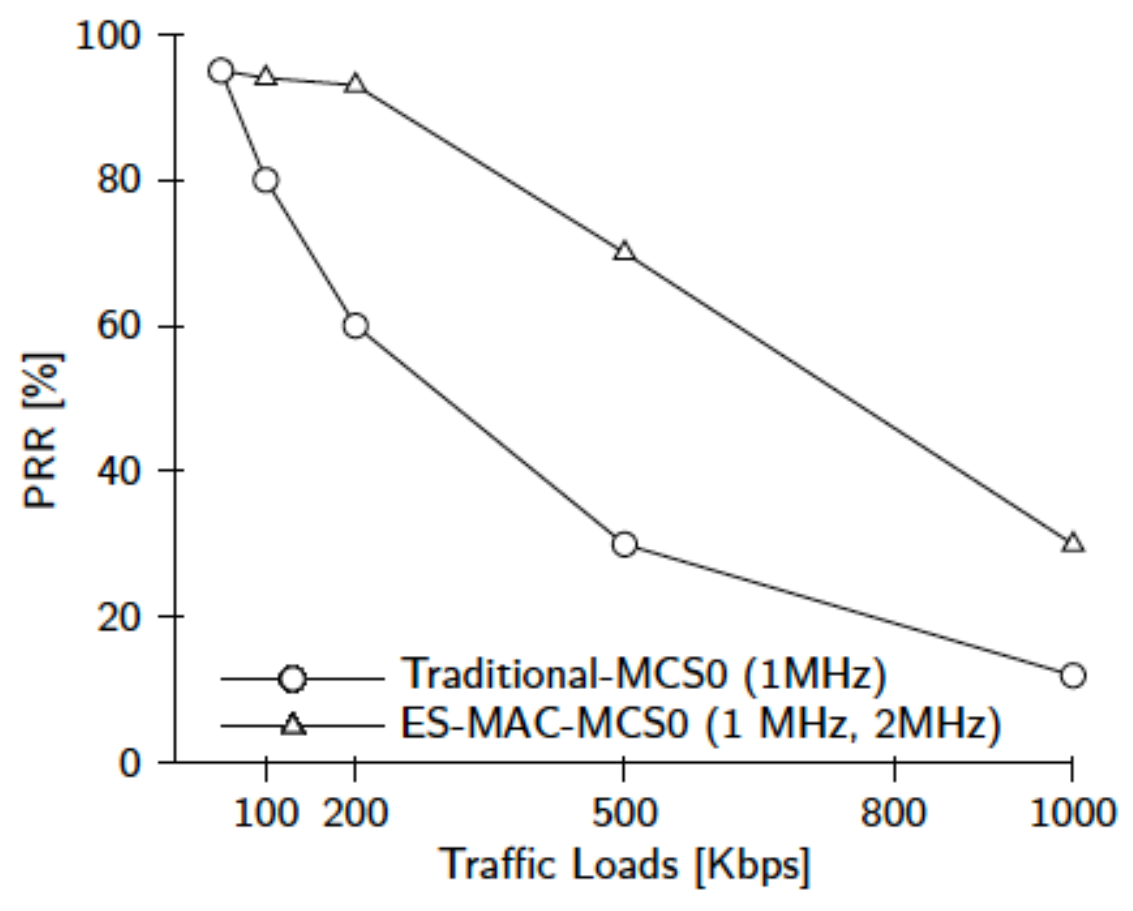

Figure 12

Packet received ratio with dynamic load scenarios 


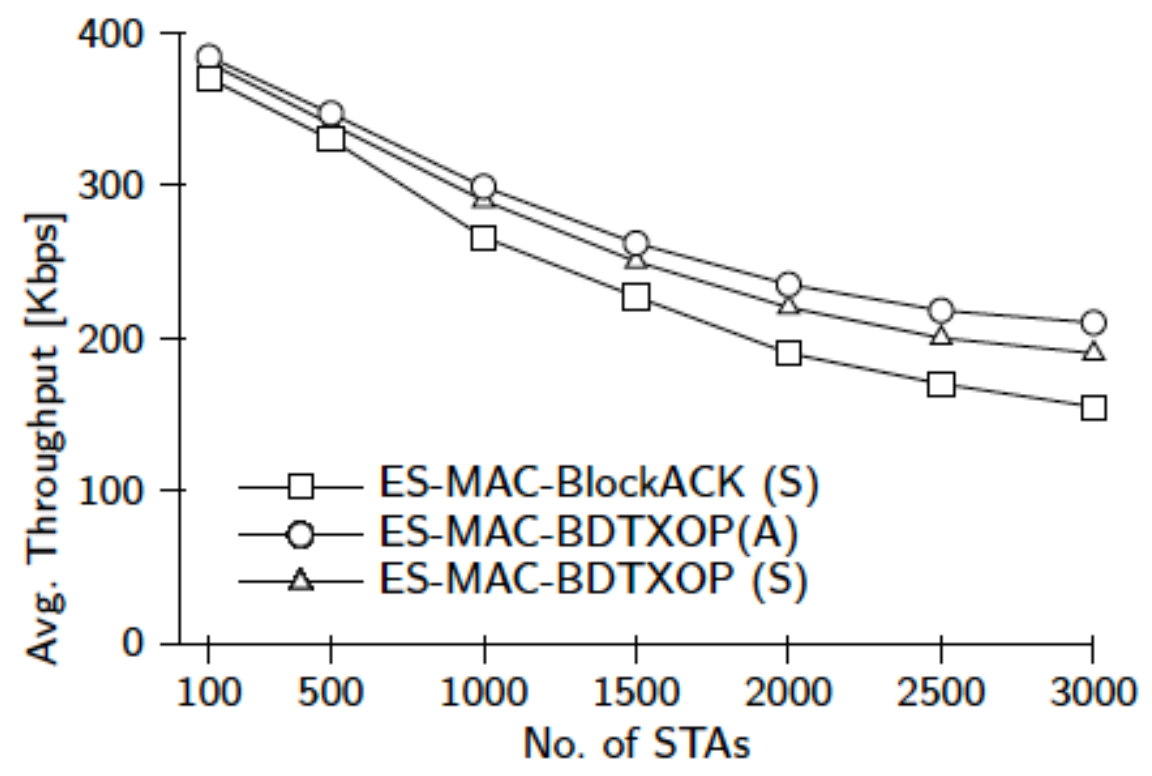

Figure 13

Performance of the TXOP-based channel access mechanism

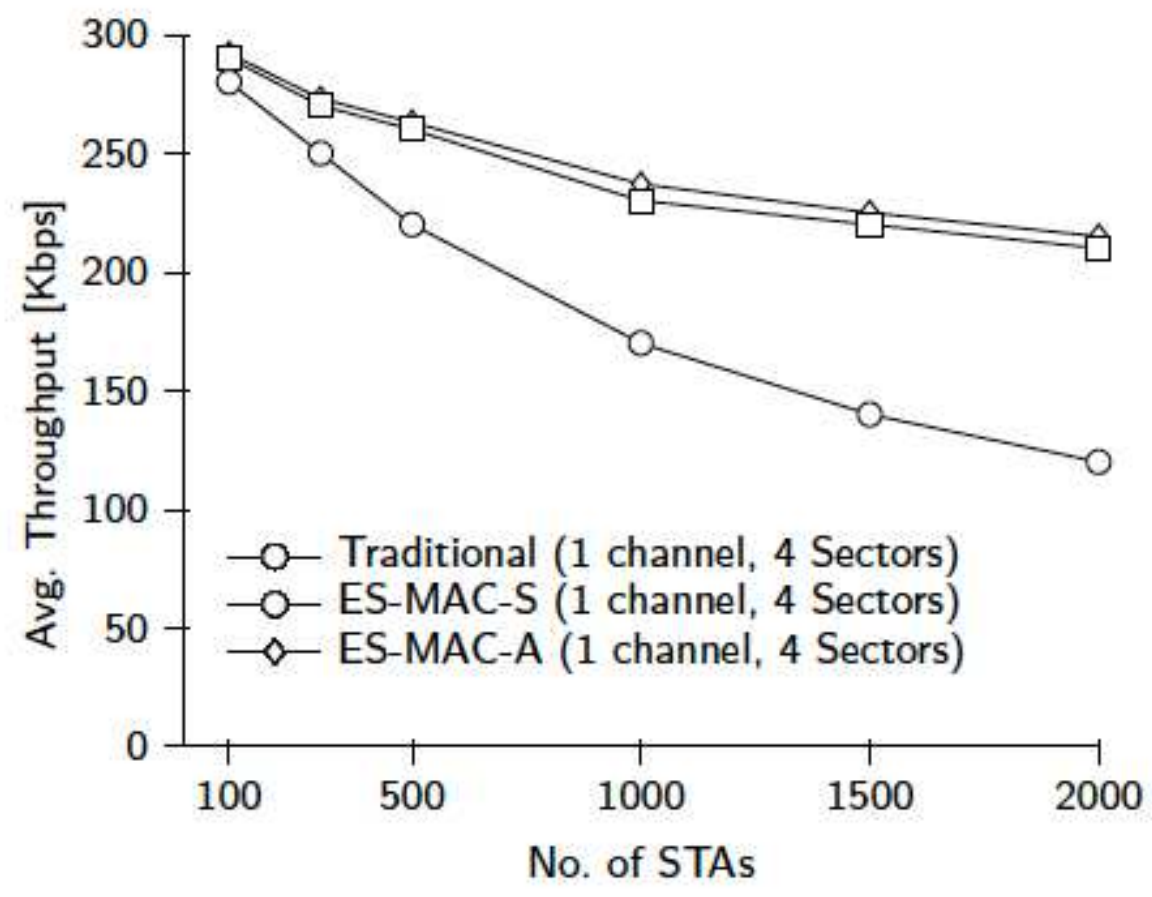

Figure 14

Overall simulation and theoretical throughput performance 